KEK-TH-1024

hep-th/0506240

\title{
Marginal Deformations and Classical Solutions in Open Superstring Field Theory
}

\author{
Isao Kishimoto $^{1, *}$ and Tomohiko TAKAhashi ${ }^{2, \dagger}$ \\ ${ }^{1}$ High Energy Accelerator Research Organization (KEK), \\ Tsukuba, Ibaraki 305-0801, Japan \\ ${ }^{2}$ Department of Physics, Nara Women's University, \\ Nara 630-8506, Japan
}

June, 2005

\begin{abstract}
We construct a class of classical solutions in the Berkovits' open superstring field theory. The resulting solutions correspond to marginal boundary deformations in conformal field theory. The vacuum energy vanishes exactly for the solutions. Investigating the theory expanded around one of the solutions, we find that it reflects the effect of background Wilson lines. The solution has a well-defined Fock space expression and it is invariant under space-time supersymmetry transformation.
\end{abstract}

\footnotetext{
${ }^{*}$ E-mail address: ikishimo@post.kek.jp

${ }^{\dagger}$ E-mail address: tomo@asuka.phys.nara-wu.ac.jp
} 


\section{Introduction}

String field theory is established as a framework for exploring nonperturbative structures in string theory. Motivated by Sen's conjecture [1, 2], many people studied classical solutions extensively and intriguing results were provided in bosonic open string field theory [3, 4]. In the supersymmetric case, the most promising theory is formulated in terms of the Wess-ZuminoWitten (WZW) like action proposed by Berkovits [5, 6], which has no problem with contact term divergences [7]. This open superstring field theory is a sufficient framework to elucidate nonperturbative phenomena of the Neveu-Schwarz (NS) sector. Indeed, the tachyon vacuum and kink solutions were found in the superstring field theory by using the level truncation scheme [6, 8, 9, 10, 11. On the analytical side, there are some attempts to construct exact solutions in terms of a half string formulation [12, a pregeometrical formulation [13, 14, a conjecture of vacuum superstring field theory [15, 16, 17, 18, 19] and an analogy with integrable systems [20].

In the present paper we construct analytic classical solutions in the open superstring field theory using techniques developed in bosonic open string field theory [21, 22, 23. The resulting solution consists of the identity string field, ghost fields and an operator associated with a current. Taking $\partial X(z)$ as the current, we find that the action expanded around the solution can be transformed to the original action by a string field redefinition. In the redefined theory, however, the momentum is shifted in the string field and then the classical solution can be related to a background Wilson line. Generically, we anticipate that our solutions correspond to marginal boundary deformed backgrounds as in the bosonic case.

The analytic solutions are useful for studying gauge structure in string field theory. In bosonic string field theory, the analytic solution corresponding to Wilson lines can be represented as a "locally" pure gauge, and then we find that a "locally" pure gauge configuration in string field theory corresponds to a marginal deformation in conformal field theory [21, 22, 23]. This correspondence is a natural generalization of that of low energy effective theories. Later we will see that the solution in the superstring field theory shares this feature of the bosonic theory.

Marginal deformations in string field theory were often studied using the level truncation scheme. We see that the effective potential for a marginal field becomes flatter as the truncation level is increased [24, 25, 26, 27, 28, 29], and then the vacuum energy of the analytic solution must vanish. Unfortunately, we encounter a difficulty in calculating the vacuum energy in the bosonic theory. Though the vacuum energy formally vanishes, it is given as a kind 
of indefinite quantities if we calculate it by oscillator representation [21, 22, 23]. However, we will see that the vacuum energy is to be exactly zero in the superstring field theory. This result is a characteristic feature of the supersymmetric case.

In string field theory, the gauge symmetry includes global symmetries generated by $K_{n}=$ $L_{n}-(-1)^{n} L_{-n}[30,31$. It is a typical symmetry in string field theory because the symmetry mixes various component fields and it has a non-local structure. Based on an analytical approach, we find that the Wilson line parameter in the solution is invariant under the global transformation.

Although it is hard to include the Ramond $(\mathrm{R})$ sector into the action, we have the equations of motion for both of the NS and R sectors 32. The equations of motion possess a fermionic symmetry which transforms the NS boson ( $\mathrm{R}$ fermion) to the $\mathrm{R}$ fermion (NS boson). Then we expect that the superstring field theory has space-time supersymmetry. Actually, we find that global space-time supersymmetry is realized on-shell as a part of the fermionic symmetry. We show that the solution corresponding to a Wilson line is a supersymmetric solution, namely the solution is invariant under the global space-time supersymmetry transformation.

This paper is organized as follows. In section 2 we construct an analytic classical solution in the open superstring field theory. We find that the solution can be written by a well-defined Fock space expression. After discussing the vacuum energy and the theory expanded around the solution, we relate the solution to background Wilson lines. Michishita gives a covariant action of the $\mathrm{R}$ sector by imposing a constraint equation [33]. We discuss the effect of the solution on the $\mathrm{R}$ sector in terms of the action proposed by Michishita. Moreover, we show how the solution is transformed under the global symmetry and space-time supersymmetry. In section 3 we extend the Wilson line solution to those which correspond to general marginal boundary deformations generated by supercurrents. We find that generalized solutions also have a favorite feature that the vacuum energy vanishes. We offer some comments related to our results and discuss open questions in section 4. In addition we include four appendices. We represent the identity string field by explicit oscillator expression in the large Hilbert space in appendix $\mathrm{A}$. In appendix $\mathrm{B}$ we give a different derivation of the action expanded around a general solution which is originally given in refs. [16, 12]. We use an alternative expression of the action given in ref. 34 to derive the expanded action. In appendix $\mathrm{C}$ we show that the fermionic symmetry contains global space-time supersymmetry, and give some comments on supersymmetry in the cubic superstring field theory [30] and its modified theory [35, 36]. In appendix D we construct the analytic solution in bosonic string field theory which corresponds to a general marginal boundary deformations. 


\section{Classical solutions and background Wilson lines}

The open superstring field theory action [5, 6] is given by

$$
S[\Phi]=\frac{1}{2 g^{2}}\left\langle\left\langle\left(e^{-\Phi} Q_{\mathrm{B}} e^{\Phi}\right)\left(e^{-\Phi} \eta_{0} e^{\Phi}\right)-\int_{0}^{1} d t\left(e^{-t \Phi} \partial_{t} e^{t \Phi}\right)\left\{\left(e^{-t \Phi} Q_{\mathrm{B}} e^{t \Phi}\right),\left(e^{-t \Phi} \eta_{0} e^{t \Phi}\right)\right\}\right\rangle\right\rangle
$$

where $\Phi$ denotes a string field of the $\mathrm{GSO}(+)$ NS sector which corresponds to a Grassmann even vertex operator of ghost number 0 and picture number 0 in the conformal field theory. CFT correlators $\langle\langle\cdots\rangle\rangle$ are defined in the large Hilbert space and $\{A, B\} \equiv A B+B A .^{1}$ The action is invariant under the infinitesimal gauge transformation,

$$
\delta e^{\Phi}=\left(Q_{\mathrm{B}} \delta \Lambda\right) * e^{\Phi}+e^{\Phi} *\left(\eta_{0} \delta \Lambda^{\prime}\right),
$$

where $\delta \Lambda$ and $\delta \Lambda^{\prime}$ are infinitesimal parameters. Integrating this infinitesimal form, we can obtain the finite gauge transformation $\mathrm{as}^{2}$

$$
e^{\Phi^{\prime}}=e^{Q_{\mathrm{B}} \Lambda} * e^{\Phi} * e^{\eta_{0} \Lambda^{\prime}}
$$

where $\Lambda$ and $\Lambda^{\prime}$ are finite parameters. Variating the action (2.1), we can derive the equation of motion to be

$$
\eta_{0}\left(e^{-\Phi} Q_{\mathrm{B}} e^{\Phi}\right)=0
$$

For simplicity, we mainly consider superstring field theory describing the dynamics of a D brane without Chan-Paton degrees of freedom. We single out a direction on the world volume of the brane, writing the string coordinate as $X(z, \bar{z})=(X(z)+X(\bar{z})) / 2$ and its supersymmetric partner as $\psi(z)$. Our later analysis can be easily extended to include ChanPaton indices.

\section{1 classical solutions in open superstring field theory}

In this subsection, we will show that one of the classical solutions is given by

$$
\Phi_{0}=-\tilde{V}_{L}(F) I
$$

\footnotetext{
${ }^{1}$ For details of the definition, see for instance ref. [8].

${ }^{2}$ The gauge transformation can be expressed as $e^{\Phi^{\prime}}=g * e^{\Phi} * g^{\prime}$ where $Q_{\mathrm{B}} g=\eta_{0} g^{\prime}=0$, since each of the operators, $Q_{\mathrm{B}}$ and $\eta_{0}$, has trivial cohomology in the large Hilbert space. Here, $g$ and $g^{\prime}$ are group elements in the "stringy gauge group" in superstring field theory.
} 
where $I$ is the identity string field and the operator $\tilde{V}_{L}$ is defined as ${ }^{3}$

$$
\tilde{V}_{L}(F)=\int_{C_{\text {left }}} \frac{d z}{2 \pi i} F(z) \tilde{v}(z), \quad \tilde{v}(z)=\frac{1}{\sqrt{2}} c \xi e^{-\phi} \psi(z) .
$$

Here, $C_{\text {left }}$ denotes a counter-clockwise path along a half of the unit circle, i.e., $-\pi / 2<\sigma<\pi / 2$ for $z=e^{i \sigma} . F(z)$ is a function on the unit circle $|z|=1$ satisfying $F(-1 / z)=z^{2} F(z)$ [22, 37]. ${ }^{4}$ We have to impose an additional constraint on $F(z)$ due to the reality of the string field as explained in the next subsection (see also appendix A).

First, we introduce half string operators similar to $\tilde{V}_{L}(F)$ :

$$
\begin{aligned}
C_{L}(F) & =\int_{C_{\mathrm{left}}} \frac{d z}{2 \pi i} F(z) c(z), \\
V_{L}(F) & =\int_{C_{\mathrm{left}}} \frac{d z}{2 \pi i} F(z) v(z),
\end{aligned}
$$

where $c(z)$ is the ghost field and $v(z)$ is defined as

$$
v(z)=\left[Q_{\mathrm{B}}, \tilde{v}(z)\right]=\frac{i}{2 \sqrt{\alpha^{\prime}}} c \partial X(z)+\frac{1}{\sqrt{2}} \eta e^{\phi} \psi(z) .
$$

By definition, the commutation relation $\left[Q_{\mathrm{B}}, \tilde{V}_{L}(F)\right]=V_{L}(F)$ holds. We also define the operators, $C_{R}(F), V_{R}(F)$ and $\tilde{V}_{R}(F)$ by replacing the integration path $C_{\text {left }}$ by $C_{\text {right }}$ which rotates counter-clockwise along $|z|=1$ ( $\operatorname{Re} z \leq 0)$. For these half string operators, we can derive their (anti-)commutation relations from similar procedures in refs. [22, 37]. The operator product expansions (OPEs) among local operators in the integrand are easily calculated as

$$
\begin{aligned}
v(z) \tilde{v}\left(z^{\prime}\right) & \sim \frac{1}{z-z^{\prime}} \frac{1}{2} c\left(z^{\prime}\right), \\
v(z) v\left(z^{\prime}\right) & \sim \frac{1}{z-z^{\prime}} \frac{-1}{2}\left(c \partial c\left(z^{\prime}\right)-\eta \partial \eta e^{2 \phi}\left(z^{\prime}\right)\right) .
\end{aligned}
$$

Using these OPEs, we obtain (anti-)commutation relations of these local operators on the unit circle, $|z|=1$ :

$$
\begin{aligned}
{\left[v(z), \tilde{v}\left(z^{\prime}\right)\right] } & =\frac{1}{2} c\left(z^{\prime}\right) \delta\left(z, z^{\prime}\right) \\
\left\{v(z), v\left(z^{\prime}\right)\right\} & =-\frac{1}{2}\left(c \partial c\left(z^{\prime}\right)-\eta \partial \eta e^{2 \phi}\left(z^{\prime}\right)\right) \delta\left(z, z^{\prime}\right)
\end{aligned}
$$

where the delta function is defined as $\delta\left(z, z^{\prime}\right)=\sum_{n=-\infty}^{\infty} z^{n} z^{\prime-n-1} .^{5}$

\footnotetext{
${ }^{3}$ We note that $e^{q \phi}(q$ : odd) is a fermionic operator. More precisely, we need a cocycle factor to represent statistical property of the operator.

${ }^{4}$ Under this condition, $F(z)$ cannot be a non-zero constant.

${ }^{5}$ The delta function satisfies

$$
f(z)=\oint_{\left|z^{\prime}\right|=1} \frac{d z^{\prime}}{2 \pi i} f\left(z^{\prime}\right) \delta\left(z, z^{\prime}\right)
$$


We integrate (2.12) and (2.13) to derive (anti-)commutation relations of half string operators:

$$
\begin{aligned}
{\left[V_{L}(F), \tilde{V}_{L}(G)\right] } & =\frac{1}{2} C_{L}(F G), \\
\left\{V_{L}(F), V_{L}(G)\right\} & =-\frac{1}{2}\left\{Q_{\mathrm{B}}, C_{L}(F G)\right\},
\end{aligned}
$$

where we have used $\left\{Q_{\mathrm{B}}, c(z)\right\}=c \partial c(z)-\eta \partial \eta e^{2 \phi}(z)$ in the latter equation. The similar relations hold for the right-half string operators, and other (anti-)commutation relations become zero. Here, we emphasize that these equations hold for any functions $F(z)$ and $G(z)$ defined on the unit circle, because we have only to use the equal-time (anti-)commutation relations to derive the equations. ${ }^{6}$ Namely, the functions are not necessarily to be holomorphic, though we express them as functions of a complex variable.

Next, we consider some properties of the half string operators associated with the star product and the identity string field. Suppose that two string fields $A$ and $B$ are defined as $|A\rangle=A(0)|0\rangle$ and $|B\rangle=B(0)|0\rangle$, where $A(z)$ and $B\left(z^{\prime}\right)$ are conformal fields on the unit discs $|z| \leq 1$ and $\left|z^{\prime}\right| \leq 1$, respectively. The star product $A * B$ is defined in terms of the gluing Riemann surface by the identification $z z^{\prime}=-1 \quad(|z|=1, \quad \operatorname{Re} z \leq 0)$ [38, 39]. Accordingly, it follows that

$$
(\sigma(z) A) * B=(-1)^{|\sigma||A|} A *\left(\left(z^{\prime 2}\right)^{h} \sigma\left(z^{\prime}\right) B\right) \quad\left(z z^{\prime}=-1, \quad|z|=1, \quad \operatorname{Re} z \leq 0\right)
$$

where $\sigma(z)$ is a primary field with dimension $h$, and $|\alpha|$ denotes the statistic index defined to be $0(1)$ if $\alpha$ is a bosonic (fermionic) operator. Multiplying a function $F(z)$ which satisfies $F(-1 / z)=\left(z^{2}\right)^{1-h} F(z)^{7}$ to both sides of (2.19) and integrating it along the path $|z|=$ $1(\operatorname{Re} z \leq 0)$, we can find the generic formula [22, 37]

$$
\left(\Sigma_{R}(F) A\right) * B=-(-1)^{|\sigma||A|} A *\left(\Sigma_{L}(F) B\right),
$$

where the function $f(z)$ is square integrable on the unit circle $|z|=1\left(f \in L^{2}\right)$. Moreover, for any $f, g \in L^{2}$, the delta function enjoys the properties,

$$
\begin{aligned}
& \int_{C_{\text {left } / \text { right }}} \frac{d z}{2 \pi i} \int_{C_{\text {left } / \text { right }}} \frac{d z^{\prime}}{2 \pi i} f(z) g\left(z^{\prime}\right) \delta\left(z, z^{\prime}\right)=\int_{C_{\text {left } / \text { right }}} \frac{d z}{2 \pi i} f(z) g(z), \\
& \int_{C_{\text {left }}} \frac{d z}{2 \pi i} \int_{C_{\text {right }}} \frac{d z^{\prime}}{2 \pi i} f(z) g\left(z^{\prime}\right) \delta\left(z, z^{\prime}\right)=0,
\end{aligned}
$$

which are necessary for derivation of (2.17) and (2.18).

${ }^{6}$ To derive (2.17) and (2.18), it is sufficient for $F$ and $G$ to be square integrable. The condition $F( \pm i)=$ $G( \pm i)=0$ is unnecessary for these (anti-)commutation relations.

${ }^{7} F(z)$ is the same as the function $F_{+}^{(-h+1)}(z)$ in ref. [22]. We note that our analysis is easily extended to the case of $F_{-}(z)$ in ref. 22. 
where the operator $\Sigma_{L(R)}$ is defined as

$$
\Sigma_{L(R)}(F)=\int_{C_{\text {left(right) }}} \frac{d z}{2 \pi i} F(z) \sigma(z) .
$$

Similarly, we can obtain a generic formula associated with the identity string field $I$ :

$$
\Sigma_{L}(F) I=-\Sigma_{R}(F) I
$$

If we choose $c(z), v(z)$ and $\tilde{v}(z)$ as the primary field, ${ }^{8}$ we can derive the following equations from the generic formulae:

$$
\begin{aligned}
\left(C_{R}\left(F_{-1}\right) A\right) * B & =-(-1)^{|A|} A *\left(C_{L}\left(F_{-1}\right) B\right), \\
\left(V_{R}\left(F_{0}\right) A\right) * B & =-(-1)^{|A|} A *\left(V_{L}\left(F_{0}\right) B\right) \\
\left(\tilde{V}_{R}\left(F_{0}\right) A\right) * B & =-A *\left(\tilde{V}_{L}\left(F_{0}\right) B\right) \\
C_{R}\left(F_{-1}\right) I & =-C_{L}\left(F_{-1}\right) I \\
V_{R}\left(F_{0}\right) I & =-V_{L}\left(F_{0}\right) I \\
\tilde{V}_{R}\left(F_{0}\right) I & =-\tilde{V}_{L}\left(F_{0}\right) I
\end{aligned}
$$

where the function $F_{h}(z)$ satisfies $F_{h}(-1 / z)=\left(z^{2}\right)^{1-h} F_{h}(z)$. Again, these equations hold if $F_{h}(z)$ is defined on the unit circle $|z|=1$. As in the case of eqs. (2.17) and (2.18), the function does not need holomorphicity. Here, the function in eq. (2.26) should behave like $F_{-1}(z) \sim \mathrm{O}\left(\left(z-z_{0}\right)^{\epsilon}\right),(\epsilon>0)$ near $z_{0}= \pm i$ in order that the state $C_{L(R)}\left(F_{-1}\right) I$ has a welldefined Fock space expression. Because the ghost field $c(z)$ has a single pole at $z= \pm i$ on the identity state as seen in the next subsection [21, 22, 40]. This condition is assured by imposing $F_{-1}( \pm i)=0$ if the function is expandable in a Taylor series. ${ }^{9}$

Now, it can be easily shown that $\Phi_{0}$ given by (2.5) is a classical solution:

$$
\begin{aligned}
e^{-\Phi_{0}} * Q_{\mathrm{B}} e^{\Phi_{0}} & =e^{\tilde{V}_{L}(F)} Q_{\mathrm{B}} e^{-\tilde{V}_{L}(F)} I \\
& =Q_{\mathrm{B}} I+\left[\tilde{V}_{L}(F), Q_{\mathrm{B}}\right] I+\frac{1}{2 !}\left[\tilde{V}_{L}(F),\left[\tilde{V}_{L}(F), Q_{\mathrm{B}}\right]\right] I+\cdots \\
& =-V_{L}(F) I+\frac{1}{4} C_{L}\left(F^{2}\right) I
\end{aligned}
$$

where we have used (2.28) and $\left[\tilde{V}_{L}(F), \tilde{V}_{R}(F)\right]=0$ in the first equality, and eq. (2.17) in the last equality. We should note that the state $C_{L}\left(F^{2}\right) I$ is well-defined because $F^{2}( \pm i)=0$ due to $F(-1 / z)=z^{2} F(z)$. The $\xi$ zero mode is not contained in both operators $V_{L}(F)$ and $C_{L}\left(F^{2}\right)$ and the identity string field satisfies $\eta_{0} I=0$. As a result, we find that $\eta_{0}\left(e^{-\Phi_{0}} * Q_{\mathrm{B}} e^{\Phi_{0}}\right)=0$ and then $\Phi_{0}$ is a solution in open superstring field theory.

\footnotetext{
${ }^{8}$ The dimensions of $c(z), v(z)$ and $\tilde{v}(z)$ are $-1,0$ and 0 , respectively.

${ }^{9}$ Actually, we can expand the function as $F_{-1}(z)=O\left(\left(z-z_{0}\right)^{2}\right)$ if $F_{-1}( \pm i)=0$ holds.
} 


\section{$2.2 \quad$ Fock space expressions}

The operator $\tilde{V}_{L}(F)$ in the solution can be expressed in terms of integration with respect to $\sigma\left(z=e^{i \sigma}\right.$ on the contour $\left.C_{\text {left }}\right)$ :

$$
\tilde{V}_{L}(F)=\int_{-\frac{\pi}{2}}^{\frac{\pi}{2}} \frac{d \sigma}{2 \pi} \frac{e^{i \sigma} F\left(e^{i \sigma}\right)}{\sqrt{2}} c(i \sigma) \xi(i \sigma): e^{-\phi(i \sigma)}: \psi(i \sigma),
$$

where operators in the integrand are given by oscillator expansions:

$$
c(i \sigma)=\sum_{n \in \mathbf{Z}} c_{n} e^{-i n \sigma}, \quad \xi(i \sigma)=\sum_{n \in \mathbf{Z}} \xi_{n} e^{-i n \sigma}, \quad \psi(i \sigma)=\sum_{r \in \mathbf{Z}+\frac{1}{2}} \psi_{r} e^{-i r \sigma}
$$

and $: e^{-\phi(i \sigma)}:$ is expanded in eq. (A.23). Using formulae: eqs. (A.17), (A.18) and (A.19) and computing straightforwardly, they are expressed on the identity state $|I\rangle$ as

$$
\begin{aligned}
& c(i \sigma)|I\rangle=\left[c_{1}(2 \cos \sigma)^{-1}+c_{0} i \tan \sigma+c_{-1}\left(1+(2 \cos \sigma)^{-1}\right)\right. \\
& \left.+2 \sum_{k \geq 1}\left(c_{-2 k} i \sin 2 k \sigma+c_{-(2 k+1)} \cos (2 k+1) \sigma\right)\right]|I\rangle \\
& \xi(i \sigma)|I\rangle=\left[\xi_{0}+2 \sum_{k \geq 1}\left(\xi_{-2 k} \cos 2 k \sigma+\xi_{-(2 k-1)} i \sin (2 k-1) \sigma\right)\right]|I\rangle, \\
& : e^{-\phi(i \sigma)}:|I\rangle=(2 \cos \sigma)^{\frac{1}{2}} e^{\sum_{k \geq 1}\left(\frac{\cos 2 k \sigma}{k} j_{-2 k}+\frac{2 i \sin (2 k-1) \sigma}{2 k-1} j_{-(2 k-1)}\right)} e^{-\hat{\phi}_{0}}|I\rangle \text {, } \\
& \psi(i \sigma)|I\rangle=\sum_{r, s \geq 1 / 2}\left(\delta_{r, s} e^{i s \sigma}+e^{-i r \sigma} I_{r s}\right) \psi_{-s}|I\rangle \\
& =\sum_{n=0}^{\infty}(2 \cos \sigma)^{\frac{1}{2}} \oint_{0} \frac{d z}{2 \pi i} z^{-n-1} \frac{\sqrt{1+z^{2}}}{\left(1-e^{i \sigma} z\right)\left(1+e^{-i \sigma} z\right)} \psi_{-\left(n+\frac{1}{2}\right)}|I\rangle \\
& =\frac{\sqrt{2}}{(\cos \sigma)^{\frac{1}{2}}} \sum_{k=0}^{\infty}\left[\psi_{-\left(2 k+\frac{1}{2}\right)} \sum_{m=0}^{k} \frac{(-1)^{k-m+1}(2(k-m)) !}{2^{2(k-m)}((k-m) !)^{2}(2(k-m)-1)} \cos (2 m+1) \sigma\right. \\
& \left.+\psi_{-\left(2 k+\frac{3}{2}\right)} \sum_{m=0}^{k} \frac{(-1)^{k-m+1}(2(k-m)) !}{2^{2(k-m)}((k-m) !)^{2}(2(k-m)-1)} i \sin 2(m+1) \sigma\right]|I\rangle \text {. }
\end{aligned}
$$

In computing $\psi(i \sigma)|I\rangle$, we have used a relation for the Neumann coefficients:

$$
\sum_{r, s \geq \frac{1}{2}} I_{r s} y^{r-\frac{1}{2}} z^{s-\frac{1}{2}}=\frac{\left(h_{I}^{\prime}(y)\right)^{\frac{1}{2}}\left(h_{I}^{\prime}(z)\right)^{\frac{1}{2}}}{h_{I}(y)-h_{I}(z)}-\frac{1}{y-z}=\frac{\sqrt{1+y^{2}} \sqrt{1+z^{2}}}{(y-z)(1+y z)}-\frac{1}{y-z},
$$

where $h_{I}(z)=2 z /\left(1-z^{2}\right)$ is the conformal map for the identity string field (see appendix $\mathrm{A}$ ). Using the above expressions and the reality $\mathrm{bpz}(|I\rangle)=(|I\rangle)^{\dagger}$, we find a relation between the $\mathrm{BPZ}$ and hermitian conjugations:

$$
\operatorname{bpz}\left(c(i \sigma) \xi(i \sigma): e^{-\phi(i \sigma)}: \psi(i \sigma)|I\rangle\right)=-\left(c(i \sigma) \xi(i \sigma): e^{-\phi(i \sigma)}: \psi(i \sigma)|I\rangle\right)^{\dagger}
$$


where we take a convention: $c_{n}^{\dagger}=c_{-n}, \xi_{n}^{\dagger}=-\xi_{-n}, j_{n}^{\dagger}=-j_{-n}-2 \delta_{n, 0}, \psi_{r}^{\dagger}=\psi_{-r}$. As a result, the reality condition for our solution $\operatorname{bpz}\left(\left|\Phi_{0}\right\rangle\right)=\left(\left|\Phi_{0}\right\rangle\right)^{\dagger} \operatorname{imposes}\left(e^{i \sigma} F\left(e^{i \sigma}\right)\right)^{*}=-e^{i \sigma} F\left(e^{i \sigma}\right)$ for the coefficient function in the integrand of (2.32), which is expanded as

$$
\begin{aligned}
e^{i \sigma} F\left(e^{i \sigma}\right) & =\sum_{n \in \mathbf{Z}} F_{n} e^{-i n \sigma}=\sum_{n \geq 1} F_{-n}\left(e^{i n \sigma}-(-1)^{n} e^{-i n \sigma}\right) \\
& =2 \sum_{k=1}^{\infty}\left(F_{-2 k} i \sin 2 k \sigma+F_{-(2 k-1)} \cos (2 k-1) \sigma\right) .
\end{aligned}
$$

Here we have used $F_{n}=-(-1)^{n} F_{-n}$ in the second equality, which follows from the condition $F(-1 / z)=z^{2} F(z)$ for the classical solution. The reality condition implies that $F_{-2 k}$ should be real and $F_{-(2 k-1)}$ should be pure imaginary.

Putting the above expansions together, we obtain the explicit Fock space expression of the classical solution as follows:

$$
\begin{aligned}
\left|\Phi_{0}\right\rangle=-\tilde{V}_{L}(F)|I\rangle & \sqrt{2} \sum_{n=1}^{\infty} \int_{-\frac{\pi}{2}}^{\frac{\pi}{2}} \frac{d \sigma}{\pi}\left(F_{-2 n} i \sin 2 n \sigma+F_{-(2 n-1)} \cos (2 n-1) \sigma\right) \\
& \times\left[c_{1}(2 \cos \sigma)^{-1}+c_{0} i \tan \sigma+c_{-1}\left(1+(2 \cos \sigma)^{-1}\right)\right. \\
& \left.\quad+2 \sum_{m \geq 1}\left(c_{-2 m} i \sin 2 m \sigma+c_{-(2 m+1)} \cos (2 m+1) \sigma\right)\right] \\
& \times\left[\xi_{0}+2 \sum_{l \geq 1}\left(\xi_{-2 l} \cos 2 l \sigma+\xi_{-(2 l-1)} i \sin (2 l-1) \sigma\right)\right] \\
& \times \exp \left[\sum_{p \geq 1}\left(\frac{\cos 2 p \sigma}{p} j_{-2 p}+\frac{2 i \sin (2 p-1) \sigma}{2 p-1} j_{-(2 p-1)}\right)\right] e^{-\hat{\phi}_{0}} \\
& \times \sum_{k=0}^{\infty}\left[\psi_{-\left(2 k+\frac{1}{2}\right)} \sum_{q=0}^{k} \frac{(-1)^{k-q}(2(k-q)) !}{2^{2(k-q)((k-q) !)^{2}(2(k-q)-1)} \cos (2 q+1) \sigma}\right. \\
& \left.\quad+\psi_{-\left(2 k+\frac{3}{2}\right)} \sum_{q=0}^{k} \frac{(-1)^{k-q}(2(k-q)) !}{2^{2(k-q)}((k-q) !)^{2}(2(k-q)-1)} i \sin 2(q+1) \sigma\right]|I\rangle .
\end{aligned}
$$

In particular, the integration with respect to $\sigma$ gives finite coefficients for each term of the form $F_{-n} c_{-m} \xi_{-l} j_{-p_{1}}^{n_{1}} \cdots j_{-p_{N}}^{n_{N}} \psi_{-s}|I\rangle$ because both $\int_{-\frac{\pi}{2}}^{\frac{\pi}{2}} \frac{d \sigma}{2 \pi}\left|\frac{\sin 2 k \sigma}{\cos \sigma}\right|$ and $\int_{-\frac{\pi}{2}}^{\frac{\pi}{2}} \frac{d \sigma}{2 \pi}\left|\frac{\cos (2 k-1) \sigma}{\cos \sigma}\right|,{ }^{10}$ in which the numerators and the denominators come from the coefficients $e^{i \sigma} F\left(e^{i \sigma}\right)$ and zero mode of ghost $c$ (i.e., $\left.c_{1}, c_{0}, c_{-1}\right)$ respectively, are finite. We note that a factor $(\cos \sigma)^{-\frac{1}{2}}$ in (2.37) is canceled

\footnotetext{
${ }^{10}$ Notice that coefficients of each term can be estimated as $M\left|\int_{-\frac{\pi}{2}}^{\frac{\pi}{2}} \frac{d \sigma}{2 \pi} \frac{\sin 2 k \sigma}{\cos \sigma} \sin ^{m_{1}} n_{1} \sigma \cdots \cos ^{p_{1}} q_{1} \sigma \cdots\right|<$ $M \int_{-\frac{\pi}{2}}^{\frac{\pi}{2}} \frac{d \sigma}{2 \pi}\left|\frac{\sin 2 k \sigma}{\cos \sigma}\right|$ or $M^{\prime}\left|\int_{-\frac{\pi}{2}}^{\frac{\pi}{2}} \frac{d \sigma}{2 \pi} \frac{\cos (2 k-1) \sigma}{\cos \sigma} \sin ^{m_{1}^{\prime}} n_{1}^{\prime} \sigma \cdots \cos ^{p_{1}^{\prime}} q_{1}^{\prime} \sigma \cdots\right|<M^{\prime} \int_{-\frac{\pi}{2}}^{\frac{\pi}{2}} \frac{d \sigma}{2 \pi}\left|\frac{\cos (2 k-1) \sigma}{\cos \sigma}\right|$, where $M, M^{\prime}$ are some finite positive constants.
} 
by a factor $(\cos \sigma)^{\frac{1}{2}}$ in (2.36) . Therefore, one can construct well-behaved solutions in the sense that coefficients of all modes in the Fock space become finite by taking appropriate $F(z)$. A sufficient condition is that only finite number of $F_{-n} \mathrm{~s}$ have nonzero value.

More concretely, the lowest few terms of the solution are computed as

$$
\begin{aligned}
\left|\Phi_{0}\right\rangle= & -\frac{\sqrt{2}}{\pi}\left(\sum_{n=1}^{\infty} \frac{(-1)^{n} F_{-(2 n-1)}}{2 n-1} c_{1} \xi_{0} \psi_{-\frac{1}{2}}\right. \\
& \left.+\sum_{n=1}^{\infty} \frac{(-1)^{n} 4 n F_{-2 n}}{4 n^{2}-1}\left(\left(c_{0} \xi_{0}+c_{1} \xi_{-1}+c_{1} \xi_{0} j_{-1}\right) \psi_{-\frac{1}{2}}+c_{1} \xi_{0} \psi_{-\frac{3}{2}}\right)+\cdots\right) e^{-\hat{\phi}_{0}}|I\rangle
\end{aligned}
$$

In the above explicit expression, the first term implies the condensation of the massless vector field because it is expanded as $c_{1} \xi_{0} \psi e^{-\hat{\phi}_{0}}|I\rangle \sim-c \xi e^{-\phi} \psi(0)|0\rangle+\cdots$ and $c \xi e^{-\phi} \psi$ is the vertex operator for massless vector with zero momentum [41]. This coefficient constant for the lowest level can be rewritten as $-\frac{\sqrt{2}}{\pi} \sum_{n=1}^{\infty} \frac{(-1)^{n} F_{-(2 n-1)}}{2 n-1}=\int_{C_{\text {left }}} \frac{d z}{2 \pi i} \frac{F(z)}{\sqrt{2}}$.

\section{3 background Wilson lines}

We found that the classical solution involves the condensation of the massless vector field. This result suggests that our solution is related to a background Wilson line. In this subsection, we will discuss the vacuum energy of the classical solution, the theory expanded around the solution, and other characteristic features of the solution. Accordingly, we will show that the solution corresponds to a background Wilson line.

In order to evaluate the vacuum energy, it is convenient to use an alternative expression for the action:

$$
\begin{aligned}
S[\Phi] & =-\frac{1}{g^{2}} \int_{0}^{1} d t\left\langle\left\langle\left(\eta_{0} e^{-t \Phi} \partial_{t} e^{t \Phi}\right)\left(e^{-t \Phi} Q_{\mathrm{B}} e^{t \Phi}\right)\right\rangle\right\rangle . \\
& =-\frac{1}{g^{2}} \int_{0}^{1} d t\left\langle\left\langle\left(\eta_{0} \Phi\right)\left(e^{-t \Phi} Q_{\mathrm{B}} e^{t \Phi}\right)\right\rangle\right\rangle .
\end{aligned}
$$

The equivalence of the actions (2.1) and (2.43) is proved in ref. 34. In general, the state $\eta_{0} \Phi$ has no $\xi$ zero mode. For the solution (2.5), it is easily seen that

$$
e^{-t \Phi_{0}} * Q_{\mathrm{B}} e^{t \Phi_{0}}=-t V_{L}(F) I+\frac{t^{2}}{4} C_{L}\left(F^{2}\right) I,
$$

and then the state $e^{-t \Phi_{0}} * Q_{\mathrm{B}} e^{t \Phi_{0}}$ also does not contain the $\xi$ zero mode. As a result, we find that the integrand in (2.43) becomes zero for the classical solution since there is no $\xi$ zero mode in the correlation function of the integrand. ${ }^{11}$ Hence we confirm that the vacuum energy of the solution vanishes due to the ghost charge non-conservation in the large Hilbert space.

\footnotetext{
${ }^{11}$ In the large Hilbert space, correlation functions are normalized as to be $\left\langle\left\langle c \partial c \partial^{2} c \xi e^{-2 \phi}\right\rangle\right\rangle \neq 0$.
} 
Let us consider the expansion of the string field around the solution (2.5). Generally, if we expand the string field $\Phi$ around a classical solution $\Phi_{0}$ as $e^{\Phi}=e^{\Phi_{0}} e^{\Phi^{\prime}}$, the action becomes

$$
\begin{aligned}
S[\Phi]= & S\left[\Phi_{0}\right]+S^{\prime}\left[\Phi^{\prime}\right] \\
S^{\prime}\left[\Phi^{\prime}\right]= & \frac{1}{2 g^{2}}\left\langle\left\langle\left(e^{-\Phi^{\prime}} Q_{\mathrm{B}}^{\prime} e^{\Phi^{\prime}}\right)\left(e^{-\Phi^{\prime}} \eta_{0} e^{\Phi^{\prime}}\right)\right.\right. \\
& \left.\left.-\int_{0}^{1} d t\left(e^{-t \Phi^{\prime}} \partial_{t} e^{t \Phi^{\prime}}\right)\left\{\left(e^{-t \Phi^{\prime}} Q_{\mathrm{B}}^{\prime} e^{t \Phi^{\prime}}\right),\left(e^{-t \Phi^{\prime}} \eta_{0} e^{t \Phi^{\prime}}\right)\right\}\right\rangle\right)
\end{aligned}
$$

where $S\left[\Phi_{0}\right]$ corresponds to the vacuum energy and $S^{\prime}\left[\Phi^{\prime}\right]$ has the same form as the original action (2.1) except the kinetic operator $Q_{\mathrm{B}}^{\prime}$, which is defined as

$$
Q_{\mathrm{B}}^{\prime} \Psi=Q_{\mathrm{B}} \Psi+A_{0} * \Psi-(-1)^{|\Psi|} \Psi * A_{0}, \quad A_{0}=e^{-\Phi_{0}} * Q_{\mathrm{B}} e^{\Phi_{0}} \quad \text { for }{ }^{\forall} \Psi
$$

A proof is given in appendix B] For the new BRS charge, nilpotency holds automatically but $\left\{Q_{\mathrm{B}}^{\prime}, \eta_{0}\right\}=0$ is satisfied owing to the equation of motion, $\eta_{0} A_{0}=0$. For the solution (2.5), we find $S\left[\Phi_{0}\right]=0$ as evaluated above. Substituting (2.5) into (2.48) and using (2.23), (2.24), (2.26), (2.27) and (2.31), we can write the new BRS charge as ${ }^{12}$

$$
Q_{\mathrm{B}}^{\prime}=Q_{\mathrm{B}}-\left(V_{L}(F)+V_{R}(F)\right)+\frac{1}{4}\left(C_{L}\left(F^{2}\right)+C_{R}\left(F^{2}\right)\right) .
$$

Using (2.17), the new BRS charge is rewritten as a similarity transformation from the original BRS charge:

$$
Q_{\mathrm{B}}^{\prime}=e^{\tilde{V}_{L}(F)+\tilde{V}_{R}(F)} Q_{\mathrm{B}} e^{-\tilde{V}_{L}(F)-\tilde{V}_{R}(F)}
$$

Here, we introduce the following half string operators,

$$
\begin{aligned}
& X_{L(R)}(F)=\int_{C_{\text {left(right) }}} \frac{d z}{2 \pi i} F(z) X(z), \\
& \Omega_{L(R)}(F)=\int_{C_{\text {left(right) }}} \frac{d z}{2 \pi i} F(z) i c \xi \partial \xi e^{-2 \phi} X(z) .
\end{aligned}
$$

Using a similar procedure in the previous subsection, we can obtain (anti-)commutation relations between these operators in terms of their OPEs:

$$
\begin{aligned}
{\left[X_{L(R)}(F), V_{L(R)}(F)\right] } & =i \sqrt{\alpha^{\prime}} C_{L(R)}\left(F^{2}\right), \\
{\left[Q_{\mathrm{B}}, X_{L(R)}(F)\right] } & =-i 2 \sqrt{\alpha^{\prime}} V_{L(R)}(F), \\
\left\{Q_{\mathrm{B}}, \Omega_{L(R)}(F)\right\} & =2 \sqrt{\alpha^{\prime}} \tilde{V}_{L(R)}(F)-i X_{L(R)}(F) .
\end{aligned}
$$

\footnotetext{
${ }^{12}$ We can check nilpotency of the new BRS charge in terms of (2.18).
} 
If $F(z)$ satisfies $F(-1 / z)=z^{2} F(z)$, it follows from (2.20) and (2.22) that

$$
\begin{aligned}
& \left(X_{R}(F) A\right) * B=-A *\left(X_{L}(F) B\right), \quad X_{R}(F) I=-X_{L}(F) I, \\
& \left(\Omega_{R}(F) A\right) * B=-(-1)^{|A|} A *\left(\Omega_{L}(F) B\right), \quad \Omega_{R}(F) I=-\Omega_{L}(F) I .
\end{aligned}
$$

Precisely speaking, $X(z)$ is not a primary field and we can not apply the formula (2.20) for the case that $\sigma(z)=X(z)$. However, it is directly shown that the equation (2.19) holds for $X(z)$ [42, 43, 44, and then we can derive the same formula in which $X(z)$ behaves like a primary field with dimension 0 on the string vertex. The same holds for the formula associated with the identity string field.

In the theory expanded around the solution (2.5), we redefine the string field $\Phi^{\prime}$ as

$$
\begin{aligned}
\Phi^{\prime} & =\exp \left(\frac{i}{2 \sqrt{\alpha^{\prime}}} X_{L}(F) I\right) * \Phi^{\prime \prime} * \exp \left(-\frac{i}{2 \sqrt{\alpha^{\prime}}} X_{L}(F) I\right) \\
& =\exp \left(\frac{i}{2 \sqrt{\alpha^{\prime}}}\left(X_{L}(F)+X_{R}(F)\right)\right) \Phi^{\prime \prime} .
\end{aligned}
$$

Under this redefinition, the action of $\Phi^{\prime}$ is transformed to the exactly same form as the original action, because $Q_{\mathrm{B}}^{\prime}$ is transformed to $Q_{\mathrm{B}}$ :

$$
Q_{\mathrm{B}}=\exp \left(-\frac{i}{2 \sqrt{\alpha^{\prime}}}\left(X_{L}(F)+X_{R}(F)\right)\right) Q_{\mathrm{B}}^{\prime} \exp \left(\frac{i}{2 \sqrt{\alpha^{\prime}}}\left(X_{L}(F)+X_{R}(F)\right)\right),
$$

where use has been made of (2.53) and (2.54). This equivalence between the original and expanded actions suggests that $\Phi_{0}$ is a pure gauge solution. Actually, we can represent the solution as a pure gauge form by using (2.55), (2.56) and (2.57):

$$
e^{\Phi_{0}}=\exp \left\{Q_{\mathrm{B}}\left(-\frac{1}{2 \sqrt{\alpha^{\prime}}} \Omega_{L}(F) I\right)\right\} * \exp \left\{\eta_{0}\left(-\frac{i}{2 \sqrt{\alpha^{\prime}}} \xi_{0} X_{L}(F) I\right)\right\} .
$$

However, this is merely a locally pure gauge expression, because the operator $X_{L}(F)$ contains the zero mode $\hat{x}$ which can not be defined globally if the direction is compactified. Hence, the classical solution turns out to be non-trivial.

In order to clarify the physical meaning of the solution, let us consider the case involving the Chan-Paton factor represented with indices $(i, j)$. The string field redefinition (2.58) can be generalized to ${ }^{13}$

$$
\begin{aligned}
\Phi_{i j}^{\prime} & =\exp \left(\frac{i}{2 \sqrt{\alpha^{\prime}}} X_{L}\left(F_{i}\right) I\right) * \Phi_{i j}^{\prime \prime} * \exp \left(-\frac{i}{2 \sqrt{\alpha^{\prime}}} X_{L}\left(F_{j}\right) I\right) \\
& =\exp \left(\frac{i}{2 \sqrt{\alpha^{\prime}}} X_{L}\left(F_{i}\right)+\frac{i}{2 \sqrt{\alpha^{\prime}}} X_{R}\left(F_{j}\right)\right) \Phi_{i j}^{\prime \prime},
\end{aligned}
$$

\footnotetext{
${ }^{13}$ The function $F_{i}(z)$ corresponds to $\lambda_{i} F(z)$ in ref. 23 .
} 
where we take no sum with respect to $(i, j)$. Noting

$$
\int_{C_{\text {left }}} \frac{d z}{2 \pi i} F(z)=-\int_{C_{\text {right }}} \frac{d z}{2 \pi i} F(z) \text { for } F(-1 / z)=z^{2} F(z),
$$

it is expanded as

$$
\Phi_{i j}^{\prime \prime}=\exp \left(-\frac{i}{2 \sqrt{\alpha^{\prime}}}\left(f_{i}-f_{j}\right) \hat{x}+\cdots\right) \Phi_{i j}^{\prime}, \quad f_{i}=\int_{C_{\text {left }}} \frac{d z}{2 \pi i} F_{i}(z),
$$

where the abbreviation on the exponent denotes nonzero mode dependence. Consequently, this string field redefinition causes shift of momentum $p \rightarrow p-\left(f_{i}-f_{j}\right) /\left(2 \sqrt{\alpha^{\prime}}\right)$ which is the same effect by background Wilson lines as shown in bosonic string field theory [21].

Thus, we conclude that the classical solution (2.5) corresponds to background Wilson lines, because the vacuum energy vanishes and the solution is represented as locally pure gauge form, and the theory expanded around the solution involves the momentum shift as expected from Wilson lines.

\subsection{Ramond sector and supersymmetry}

The action of the Ramond sector proposed in ref. [33] is:

$$
S_{F}=-\frac{1}{2 g^{2}}\left\langle\left\langle\left(Q_{\mathrm{B}} \Xi\right) e^{\Phi}\left(\eta_{0} \Psi\right) e^{-\Phi}\right\rangle\right\rangle,
$$

where $\Psi$ is a string field of the $\mathrm{GSO}(+) \mathrm{R}$ sector which has ghost number 0 and picture number 1/2. $\Xi$ carries ghost number 0 and picture number $-1 / 2$. Both $\Psi$ and $\Xi$ are Grassmann even. The total action is given by adding $S_{F}$ to the NS action (2.1), and a constraint is imposed on string fields as

$$
Q_{\mathrm{B}} \Xi=e^{\Phi}\left(\eta_{0} \Psi\right) e^{-\Phi}
$$

The total action is invariant under the infinitesimal gauge transformation

$$
\begin{aligned}
\delta e^{\Phi} & =\left(Q_{\mathrm{B}} \delta \Lambda_{0}\right) * e^{\Phi}+e^{\Phi} *\left(\eta_{0} \delta \Lambda_{1}\right) \\
\delta \Psi & =\eta_{0} \delta \Lambda_{3 / 2}+\Psi *\left(\eta_{0} \delta \Lambda_{1}\right)-\left(\eta_{0} \delta \Lambda_{1}\right) * \Psi \\
\delta \Xi & =Q_{\mathrm{B}} \delta \Lambda_{-1 / 2}+\left(Q_{\mathrm{B}} \delta \Lambda_{0}\right) * \Xi-\Xi *\left(Q_{\mathrm{B}} \delta \Lambda_{0}\right),
\end{aligned}
$$

where $\delta \Lambda_{P}$ denotes an infinitesimal parameter with the picture number $P$. The constraint (2.65) is unchanged under the transformation. 
Variating the total action, we can derive the equations of motion to be [33]

$$
\begin{aligned}
\eta_{0}\left(e^{-\Phi} Q_{\mathrm{B}} e^{\Phi}\right) & =-\frac{1}{2}\left(\eta_{0} \Psi\right) e^{-\Phi}\left(Q_{\mathrm{B}} \Xi\right) e^{\Phi}-\frac{1}{2} e^{-\Phi}\left(Q_{\mathrm{B}} \Xi\right) e^{\Phi}\left(\eta_{0} \Psi\right), \\
\eta_{0}\left(e^{-\Phi}\left(Q_{\mathrm{B}} \Xi\right) e^{\Phi}\right) & =0 \\
Q_{\mathrm{B}}\left(e^{\Phi}\left(\eta_{0} \Psi\right) e^{-\Phi}\right) & =0 .
\end{aligned}
$$

Substituting the constraint (2.65) into these equations, we can obtain the equations of motion for the NS and R sectors, $\eta_{0}\left(e^{-\Phi} Q_{\mathrm{B}} e^{\Phi}\right)=-\left(\eta_{0} \Psi\right)^{2}$ and $Q_{\mathrm{B}}\left(e^{\Phi}\left(\eta_{0} \Psi\right) e^{-\Phi}\right)=0$ [32].

Let us expand the string fields around a classical solution $(\Phi, \Psi)=\left(\Phi_{0}, 0\right)$ as $\left(e^{\Phi}, \Psi\right)=$ $\left(e^{\Phi_{0}} e^{\Phi^{\prime}}, \Psi^{\prime}\right)$. Then, the action of the $\mathrm{R}$ sector becomes

$$
S_{F}=-\frac{1}{2 g^{2}}\left\langle\left\langle\left(Q_{\mathrm{B}}^{\prime} \Xi^{\prime}\right) e^{\Phi^{\prime}}\left(\eta_{0} \Psi^{\prime}\right) e^{-\Phi^{\prime}}\right\rangle\right\rangle,
$$

and the constraint is changed to

$$
Q_{\mathrm{B}}^{\prime} \Xi^{\prime}=e^{\Phi^{\prime}}\left(\eta_{0} \Psi^{\prime}\right) e^{-\Phi^{\prime}}
$$

where $Q_{\mathrm{B}}^{\prime}$ is the new BRS operator defined as (2.48) and $\Xi$ is a superfluous string field redefined as $\Xi^{\prime}=e^{-\Phi_{0}} \Xi e^{\Phi_{0}}$. Like the NS sector, the expanded action and constraint in the R sector have the same structure as the original ones except the form of the BRS operator.

Now, we take the solution (2.5) as $\Phi_{0}$ in the above expansion. In the expanded action, we redefine the string fields as

$$
\begin{aligned}
& \Phi^{\prime}=\exp \left(\frac{i}{2 \sqrt{\alpha^{\prime}}} X_{L}(F) I\right) * \Phi^{\prime \prime} * \exp \left(-\frac{i}{2 \sqrt{\alpha^{\prime}}} X_{L}(F) I\right), \\
& \Psi^{\prime}=\exp \left(\frac{i}{2 \sqrt{\alpha^{\prime}}} X_{L}(F) I\right) * \Psi^{\prime \prime} * \exp \left(-\frac{i}{2 \sqrt{\alpha^{\prime}}} X_{L}(F) I\right), \\
& \Xi^{\prime}=\exp \left(\frac{i}{2 \sqrt{\alpha^{\prime}}} X_{L}(F) I\right) * \Xi^{\prime \prime} * \exp \left(-\frac{i}{2 \sqrt{\alpha^{\prime}}} X_{L}(F) I\right) .
\end{aligned}
$$

We can easily find that the new BRS charge is transformed to the original form in both of the total action and the constraint. As in the previous subsection, the classical solution has an effect only on the momentum in the string fields. The result indicates that the classical solution (2.5) corresponds to the background Wilson line in open superstring field theory including the NS and R sector.

Instead of using the action, we can see the effect of the classical solution in terms of the equation of motion. The original Berkovits' equations of motion are given by $\eta_{0}\left(e^{-\Phi} Q_{\mathrm{B}} e^{\Phi}\right)=$ $-\left(\eta_{0} \Psi\right)^{2}, Q_{\mathrm{B}}\left(e^{\Phi}\left(\eta_{0} \Psi\right) e^{-\Phi}\right)=0$. Expanding the equations of motion around the classical 
solution, we find that the form of the equations is unchanged but the BRS charge is changed to $Q_{\mathrm{B}}^{\prime}$ of (2.48). In the case of our solution $\Phi_{0}=-\tilde{V}_{L}(F) I, \Psi_{0}=0$ (2.5), the expanded equations of motion are transformed back to the original ones by the field redefinitions: $\Phi^{\prime}=$ $e^{\frac{i}{2 \sqrt{\alpha^{\prime}}} X_{L}(F) I} * \Phi^{\prime \prime} * e^{-\frac{i}{2 \sqrt{\alpha^{\prime}}} X_{L}(F) I}, \Psi^{\prime}=e^{\frac{i}{2 \sqrt{\alpha^{\prime}}} X_{L}(F) I} * \Psi^{\prime \prime} * e^{-\frac{i}{2 \sqrt{\alpha^{\prime}}} X_{L}(F) I}$. This redefinition reflects the effect of the Wilson lines.

The equations of motion for the NS and R sectors have a fermionic gauge symmetry as follows [32]:

$$
\begin{aligned}
\delta e^{\Phi} & =-e^{\Phi} *\left(\eta_{0} \Psi * \Lambda_{1 / 2}+\Lambda_{1 / 2} * \eta_{0} \Psi\right), \\
\delta \Psi & =Q_{\mathrm{B}} \Lambda_{1 / 2}+e^{-\Phi} Q_{\mathrm{B}} e^{\Phi} * \Lambda_{1 / 2}+\Lambda_{1 / 2} * e^{-\Phi} Q_{\mathrm{B}} e^{\Phi},
\end{aligned}
$$

where $\Lambda_{1 / 2}$ is a Grassmann odd parameter with the picture number $1 / 2$. This may include a global space-time supersymmetry, since the NS (R) string field is transformed to the R (NS) sector under the transformation. Actually, we can find the supersymmetry if we formally set a transformation parameter as

$$
\Lambda_{1 / 2}=\Omega(\epsilon)=\epsilon_{\alpha} \int_{C_{\text {left }}} \frac{d z}{2 \pi i} \xi S_{(-1 / 2)}^{\alpha}(z) I,
$$

where $S_{(-1 / 2)}^{\alpha}$ is a $\mathrm{GSO}(+)$ spin field with $\phi$-charge $-1 / 2$ and positive chirality and $\epsilon_{\alpha}$ is a fermionic constant. We give the details of the supersymmetry in appendix C. Substituting (2.79) into eqs. (2.77) and (2.78), we can rewrite the transformation law as ${ }^{14}$

$$
\delta_{\epsilon} e^{\Phi}=-e^{\Phi} \mathcal{S}(\epsilon) \eta_{0} \Psi, \quad \delta_{\epsilon}\left(\eta_{0} \Psi\right)=\eta_{0} \mathcal{S}(\epsilon)\left(e^{-\Phi} Q_{\mathrm{B}} e^{\Phi}\right),
$$

where the operator $\mathcal{S}(\epsilon)$ is defined as

$$
\mathcal{S}(\epsilon)=\epsilon_{\alpha} \oint \frac{d z}{2 \pi i} \xi S_{(-1 / 2)}^{\alpha}(z)
$$

The operator $\mathcal{S}(\epsilon)$ is an anti-derivation with respect to the star product. Now, we apply this transformation to the Wilson line solution, namely $\Phi_{0}$ given by (2.5) and $\Psi_{0}=0$ :

$$
\begin{aligned}
\delta_{\epsilon} e^{\Phi} & =0, \\
\delta_{\epsilon}\left(\eta_{0} \Psi\right) & =\eta_{0} \mathcal{S}(\epsilon)\left(e^{-\Phi_{0}} Q_{\mathrm{B}} e^{\Phi_{0}}\right)=\epsilon_{\alpha} \oint \frac{d z}{2 \pi i} S_{(-1 / 2)}^{\alpha}(z)\left(V_{L}(F)-\frac{1}{4} C_{L}\left(F^{2}\right)\right) I \\
& =\epsilon_{\alpha} \oint \frac{d z}{2 \pi i}\left\{S_{(-1 / 2)}^{\alpha}(z), V_{L}(F)\right\} I=0,
\end{aligned}
$$

\footnotetext{
14 We do not include a contribution from the first term in 2.78): $\delta_{\mathrm{M}}\left(\eta_{0} \Psi\right) \equiv-Q_{\mathrm{B}} \eta_{0} \Omega(\epsilon)=$ $\frac{i}{2 \pi} \epsilon_{\alpha}\left(c S_{(-1 / 2)}^{\alpha}(i)-c S_{(-1 / 2)}^{\alpha}(-i)\right) I$. The elimination of this term is possible because the transformation $\delta_{\mathrm{M}}$ is a symmetry of the equations of motion: $\delta_{\mathrm{M}} f_{1}=\left\{\mathcal{O}(i) I, \eta_{0} \Psi\right\}=\mathcal{O}(i) \eta_{0} \Psi-\mathcal{O}(i) \eta_{0} \Psi=0, \quad \delta_{\mathrm{M}} f_{2}=$ $\left\{\mathcal{O}(i) I, e^{-\Phi} Q_{\mathrm{B}} e^{\Phi}\right\}=0$, where $f_{1}$ and $f_{2}$ are given in eqs. (C.3) and (C.4) respectively and $\mathcal{O}(i) \equiv$ $\frac{i}{2 \pi} \epsilon_{\alpha}\left(c S_{(-1 / 2)}^{\alpha}(i)-c S_{(-1 / 2)}^{\alpha}(-i)\right)$.
} 
where use has been made of $\left\{v(y), S_{(-1 / 2)}^{\alpha}(z)\right\}=0$. We note that $\oint \frac{d z}{2 \pi i} S_{(-1 / 2)}^{\alpha} I=0$ and $\mathcal{S}(\epsilon) I=0$ because both $S_{(-1 / 2)}^{\alpha}(z)$ and $\xi S_{(-1 / 2)}^{\alpha}(z)$ are primary fields with conformal dimension 1. This result indicates that the Wilson line solution exactly preserves all global space-time supersymmetries.

\section{5 symmetries and classical solutions}

The half integration of $F(z)$ is related to a Wilson line parameter, which should be a gauge invariant quantity of the stringy gauge group. This relation suggests that other modes of $F(z)$ are redundant degrees of freedom under the gauge symmetry. In this subsection we would like to illustrate this point, namely the function $F(z)$ can be changed by an appropriate transformation except the half integration mode.

The total action including the $\mathrm{R}$ sector is invariant under the finite gauge transformation,

$$
\begin{aligned}
e^{\Phi^{\prime}} & =e^{Q_{\mathrm{B}} \Lambda_{0}} * e^{\Phi} * e^{\eta_{0} \Lambda_{1}}, \\
\Psi^{\prime} & =e^{-\eta_{0} \Lambda_{1}} * \Psi * e^{\eta_{0} \Lambda_{1}}+\frac{1-e^{-\mathrm{ad}_{\eta_{0} \Lambda_{1}}}}{\operatorname{ad}_{\eta_{0} \Lambda_{1}}}\left(\eta_{0} \Lambda_{3 / 2}\right), \\
\Xi^{\prime} & =e^{Q_{\mathrm{B}} \Lambda_{0}} * \Xi * e^{-Q_{\mathrm{B}} \Lambda_{0}}+\frac{e^{\operatorname{ad}_{Q_{\mathrm{B}} \Lambda_{0}}-1}}{\operatorname{ad}_{Q_{\mathrm{B}} \Lambda_{0}}}\left(Q_{\mathrm{B}} \Lambda_{-1 / 2}\right) .
\end{aligned}
$$

This transformation can be obtained by performing $N$ times of the infinitesimal transformation given in eqs. (2.66), (2.67) and (2.68) and then taking the limit $N \rightarrow \infty$ :

$$
\begin{aligned}
e^{\Phi^{\prime}} & =\lim _{N \rightarrow \infty}\left(1+\frac{1}{N} Q_{\mathrm{B}} \Lambda_{0}\right)^{N} e^{\Phi}\left(1+\frac{1}{N} \eta_{0} \Lambda_{1}\right)^{N}, \\
\Psi^{\prime} & =\lim _{N \rightarrow \infty}\left[\left(1-\frac{1}{N} \operatorname{ad}_{\eta_{0} \Lambda_{1}}\right)^{N} \Psi+\sum_{k=0}^{N-1}\left(1-\frac{1}{N} \operatorname{ad}_{\eta_{0} \Lambda_{1}}\right)^{k} \frac{1}{N} \eta_{0} \Lambda_{3 / 2}\right], \\
\Xi^{\prime} & =\lim _{N \rightarrow \infty}\left[\left(1+\frac{1}{N} \operatorname{ad}_{Q_{\mathrm{B}} \Lambda_{0}}\right)^{N} \Xi+\sum_{k=0}^{N-1}\left(1+\frac{1}{N} \operatorname{ad}_{Q_{\mathrm{B}} \Lambda_{0}}\right)^{k} \frac{1}{N} Q_{\mathrm{B}} \Lambda_{-1 / 2}\right],
\end{aligned}
$$

where we set $\delta \Lambda_{P}=\Lambda_{P} / N$ in (2.66), (2.67) and (2.68) and we have used the definition $\operatorname{ad}_{X} Y \equiv[X, Y]=X * Y-Y * X$.

Substituting $Q_{\mathrm{B}} \Lambda_{0}=-\eta_{0} \Lambda_{1}=\Upsilon_{0}$ and $\Lambda_{3 / 2}=\Lambda_{-1 / 2}=0$ into (2.84), (2.85) and (2.86), we find

$$
e^{\Phi^{\prime}}=e^{\Upsilon_{0}} * e^{\Phi} * e^{-\Upsilon_{0}}, \quad \Psi^{\prime}=e^{\Upsilon_{0}} * \Psi * e^{-\Upsilon_{0}}, \quad \Xi^{\prime}=e^{\Upsilon_{0}} * \Xi * e^{-\Upsilon_{0}} .
$$

In the large Hilbert space, any state $\Upsilon_{0}$ satisfying $Q_{\mathrm{B}} \Upsilon_{0}=\eta_{0} \Upsilon_{0}=0$ can be written as $\Upsilon_{0}=Q_{\mathrm{B}} \Lambda_{0}=-\eta_{0} \Lambda_{1}$. Hence, the total action is invariant under the similarity transformation generated by $\Upsilon_{0}$ such that $Q_{\mathrm{B}} \Upsilon_{0}=\eta_{0} \Upsilon_{0}=0$. 
The BRS charge $Q_{\mathrm{B}}$ corresponds to the external derivative in the WZW theory. It is easy to show that the "pure gauge connection" $A_{Q}=e^{-\Phi} * Q_{\mathrm{B}} e^{\Phi}$ is transformed as

$$
A_{Q}^{\prime}=e^{-\eta_{0} \Lambda_{1}} * Q_{\mathrm{B}} e^{\eta_{0} \Lambda_{1}}+e^{-\eta_{0} \Lambda_{1}} * A_{Q} * e^{\eta_{0} \Lambda_{1}},
$$

under the gauge transformation. If we perform the similarity transformation (2.90) on $A_{Q}$, we find $A_{Q}^{\prime}=e^{\Upsilon_{0}} * A_{Q} * e^{-\Upsilon_{0}}$ since $Q_{\mathrm{B}} e^{\Upsilon_{0}}=0$. This transformation law allows us to interpret (2.90) as a "global transformation" [31, which is a transformation of a subgroup of the stringy gauge group.

Now, let us consider a "global transformation" generated by the parameter,

$$
\Upsilon_{0}=\mathcal{T}_{L}(f) I=\int_{C_{\text {left }}} \frac{d z}{2 \pi i} f(z) T(z) I, \quad f(-1 / z)=z^{-2} f(z),
$$

where $T(z)$ is the total energy momentum tensor. It is easily seen that $\Upsilon_{0}$ satisfies $Q_{\mathrm{B}} \Upsilon_{0}=$ $\eta_{0} \Upsilon_{0}=0$ since $\left[Q_{\mathrm{B}}, T(z)\right]=\left[\eta_{0}, T(z)\right]=0$. More explicitly, the gauge transformation parameters $\Lambda_{0}$ and $\Lambda_{1}$ satisfying $Q_{\mathrm{B}} \Lambda_{0}=-\eta_{0} \Lambda_{1}=\Upsilon_{0}$ can be written as

$$
\Lambda_{0}=U_{L}(f) I, \quad \Lambda_{1}=-\xi_{0} \mathcal{T}_{L}(f) I,
$$

where $U_{L}(f)$ is defined as ${ }^{15}$

$$
\begin{aligned}
U_{L}(f)= & \int_{C_{\text {left }}} \frac{d z}{2 \pi i} f(z) u(z) \\
u(z)= & -T^{\mathrm{m}} c \xi \partial \xi e^{-2 \phi}(z)-2 b c \partial c \xi \partial \xi e^{-2 \phi}(z)+c \partial \xi \partial^{2} \xi e^{-2 \phi}(z)-\frac{3}{2} \partial^{2} c \xi \partial \xi e^{-2 \phi}(z) \\
& -\frac{1}{2} c \xi \partial^{3} \xi e^{-2 \phi}(z)+c \xi \partial \xi\left(\frac{1}{2}(\partial \phi)^{2}+3 \partial^{2} \phi\right) e^{-2 \phi}(z)
\end{aligned}
$$

Using some properties of $\mathcal{T}_{L}(f),{ }^{16}$ we can rewrite the global transformation as

$$
\Phi^{\prime}=e^{\mathcal{T}(f)} \Phi, \quad \Psi^{\prime}=e^{\mathcal{T}(f)} \Psi, \quad \Xi^{\prime}=e^{\mathcal{T}(f)} \Xi, \quad \mathcal{T}(f)=\oint \frac{d z}{2 \pi i} f(z) T(z) .
$$

If we apply an infinitesimal transformation on the classical solution (2.5), it changes to

$$
\begin{aligned}
\Phi_{0}^{\prime} & =e^{\mathcal{T}(\epsilon)} \Phi_{0}=-\tilde{V}_{L}\left(F^{\prime}\right) I, \\
F^{\prime}(z) & =F(z)-\partial(\epsilon(z) F(z)),
\end{aligned}
$$

${ }^{15} T^{\mathrm{m}}(z)$ is the matter energy momentum tensor. We note that $\left\{Q_{\mathrm{B}}, u(z)\right\}=T(z)$.

${ }^{16}$ The operator $\mathcal{T}_{L}(f)$ satisfies

$$
\begin{aligned}
& {\left[\mathcal{T}_{L(R)}(f), \mathcal{T}_{L(R)}(g)\right]=\mathcal{T}_{L(R)}((\partial f) g-f \partial g), \quad\left[\mathcal{T}_{L}(f), \mathcal{T}_{R}(g)\right]=0,} \\
& \left(\mathcal{T}_{R}(f) A\right) * B=-A *\left(\mathcal{T}_{L}(f) B\right), \quad \mathcal{T}_{R}(f) I=-\mathcal{T}_{L}(f) I,
\end{aligned}
$$

as in the bosonic case [31. 
where we have used the commutation relation $\left[\mathcal{T}(f), \tilde{V}_{L}(F)\right]=-\tilde{V}_{L}(\partial(f F))$. We note that $F^{\prime}(z)$ satisfies $F^{\prime}(-1 / z)=z^{2} F^{\prime}(z)$. Accordingly, we find that the function form of $F(z)$ are redundant under the gauge transformation. However, we cannot change the half integration mode of $F(z)$. Indeed, we find that, from eq. (2.100),

$$
f=\int_{C_{\text {left }}} \frac{d z}{2 \pi i} F(z)=\int_{C_{\text {left }}} \frac{d z}{2 \pi i} F^{\prime}(z),
$$

where we have used $\epsilon( \pm i)=F( \pm i)=0$ due to $\epsilon(-1 / z)=z^{-2} \epsilon(z)$ and $F(-1 / z)=z^{2} F(z)$. These are consistent results with our expectation. The half integration mode of $F(z)$, which is to be a physical quantity, is invariant but other modes can be gauged away.

\section{Marginal deformations and classical solutions}

In the previous section, we have described a class of solutions which correspond to the Wilson lines. It turns out that they are based on algebra satisfied by $u(1)$ supercurrent $\mathbf{J}(z, \theta)=$ $\psi(z)+\theta \frac{i}{\sqrt{2 \alpha^{\prime}}} \partial X(z)$. From this point of view, we can use the same method to construct classical solutions of superstring field theory which correspond to more general supercurrents or marginal deformations in the context of conformal field theory. ${ }^{17}$

Here we consider a supercurrent $\mathbf{J}^{a}(z, \theta)=\psi^{a}(z)+\theta J^{a}(z)$ associated with a Lie algebra $\mathcal{G}$ in the matter sector $(a=1, \cdots, \operatorname{dim} \mathcal{G})$. In terms of component fields, we suppose that OPE is given by

$$
\begin{aligned}
\psi^{a}(y) \psi^{b}(z) & \sim \frac{1}{y-z} \frac{1}{2} \Omega^{a b} \\
J^{a}(y) \psi^{b}(z) & \sim \frac{1}{y-z} f_{c}^{a b} \psi^{c}(z) \\
J^{a}(y) J^{b}(z) & \sim \frac{1}{(y-z)^{2}} \frac{1}{2} \Omega^{a b}+\frac{1}{y-z} f_{c}^{a b} J^{c}(z),
\end{aligned}
$$

where $f_{c}^{a b}$ is the structure constant of $\mathcal{G}\left(f_{c}^{a b}=-f_{c}^{b a}, f^{a b}{ }_{d} f^{c d}{ }_{e}+f^{b c}{ }_{d} f_{e}^{a d}+f^{c a}{ }_{d} f^{b d}{ }_{e}=0\right)$ and $\Omega^{a b}$ is an invertible matrix ${ }^{18}$ which satisfies

$$
\Omega^{a b}=\Omega^{b a}, \quad f_{c}^{a b} \Omega^{c d}+f_{c}^{a d} \Omega^{c b}=0 .
$$

\footnotetext{
${ }^{17}$ As a comparison, we discuss a similar generalization in the context of the Witten's bosonic string field theory in appendix D

${ }^{18}$ In the case of semi-simple Lie algebra, we can take $\Omega^{a b}$ as the Killing form $\gamma^{a b}=f_{d}^{a c} f_{c}^{b d}$. However, we have supposed the existence of invertible $\Omega^{a b}$ in order to include the cases of non-semi-simple algebra after ref. 45.
} 
In this case, energy momentum tensor $T(z)$ and its super partner $G(z)$ are given by a general Sugawara construction [45]:

$$
\begin{aligned}
& T(z)=\Omega_{a b}:\left(J^{a} J^{b}+\partial \psi^{a} \psi^{b}\right):(z)+\frac{2}{3} \Omega_{a d} \Omega_{b e} f_{c}^{d e}:\left(J^{a}: \psi^{b} \psi^{c}:+\psi^{a}:\left(\psi^{b} J^{c}-J^{b} \psi^{c}\right):\right):(z), \\
& G(z)=2 \Omega_{a b}: J^{a} \psi^{b}:(z)+\frac{4}{3} \Omega_{a d} \Omega_{b e} f_{c}^{d e}: \psi^{a}: \psi^{b} \psi^{c}::(z)
\end{aligned}
$$

where $\Omega_{a b}$ is the inverse of $\Omega^{a b}: \Omega^{a b} \Omega_{b c}=\delta_{c}^{a}$. In fact, they satisfy the following OPEs:

$$
\begin{aligned}
T(y) \psi^{a}(z) & \sim \frac{1}{(y-z)^{2}} \frac{1}{2} \psi^{a}(z)+\frac{1}{y-z} \partial \psi^{a}(z) \\
T(y) J^{a}(z) & \sim \frac{1}{(y-z)^{2}} J^{a}(z)+\frac{1}{y-z} \partial J^{a}(z) \\
G(y) \psi^{a}(z) & \sim \frac{1}{y-z} J^{a}(z) \\
G(y) J^{a}(z) & \sim \frac{1}{(y-z)^{2}} \psi^{a}(z)+\frac{1}{y-z} \partial \psi^{a}(z), \\
T(y) T(z) & \sim \frac{c}{2} \frac{1}{(y-z)^{4}}+\frac{1}{(y-z)^{2}} 2 T(z)+\frac{1}{y-z} \partial T(z), \\
G(y) G(z) & \sim \frac{1}{3} \frac{1}{(y-z)^{3}}+\frac{1}{y-z} 2 T(z), \\
T(y) G(z) & \sim \frac{1}{(y-z)^{2}} \frac{3}{2} G(z)+\frac{1}{y-z} \partial G(z)
\end{aligned}
$$

and the central charge $c$ is given by $c=\frac{3}{2} \operatorname{dim} \mathcal{G}-f_{d}^{a c} f_{c}^{b d} \Omega_{a b}$ [45].

Let us consider the Berkovits' open superstring field theory on the above CFT background. The action has the same form as the flat one:

$$
S[\Phi]=-\frac{1}{g^{2}} \int_{0}^{1} d t\left\langle\left\langle\left(\eta_{0} \Phi\right)\left(e^{-t \Phi} Q_{\mathrm{B}} e^{t \Phi}\right)\right\rangle\right\rangle
$$

although $T(z)$ and $G(z)$ in the definition of the BRS operator ${ }^{19}$ are given by (3.5) and (3.6). The star product among string fields in the action is constructed by LPP's method [38] in terms of conformal mappings and correlators in the above CFT. In order to make $Q_{\mathrm{B}}$ nilpotent, we assume that the total central charge in the matter sector is $c=15$. With this setup, we shall show that

$$
\begin{aligned}
& \Phi_{0}=-\tilde{V}_{L}^{a}\left(F_{a}\right) I, \\
& \tilde{V}_{L}^{a}\left(F_{a}\right)=\int_{C_{\text {left }}} \frac{d z}{2 \pi i} F_{a}(z) \tilde{v}^{a}(z), \quad F_{a}(-1 / z)=z^{2} F_{a}(z),
\end{aligned}
$$

${ }^{19}$ The BRS operator is given by the matter Virasoro operators $T(z), G(z)$ and ghosts $(b, c, \phi, \xi, \eta)$ as:

$$
Q_{\mathrm{B}}=\oint \frac{d z}{2 \pi i}\left[c\left(T-\frac{1}{2}(\partial \phi)^{2}-\partial^{2} \phi+\partial \xi \eta\right)(z)+b c \partial c(z)+\eta e^{\phi} G(z)-\eta \partial \eta e^{2 \phi} b(z)\right] .
$$


is a classical solution, where the operator $\tilde{v}^{a}(z)$ is given by the lowest component of supercurrent $\mathbf{J}^{a}(z, \theta)$ with dimension $1 / 2$ and appropriate ghost part:

$$
\tilde{v}^{a}(z)=\frac{1}{\sqrt{2}} c \xi e^{-\phi} \psi^{a}(z),
$$

and $I$ is the identity string field which is the identity element with respect to the star product. Noting $\tilde{v}^{a}(z)$ is a primary field with dimension 0 and therefore satisfies

$$
\left(\tilde{V}_{L}^{a}\left(F_{a}\right) I\right) * B=-\tilde{V}_{R}^{a}\left(F_{a}\right) I * B=I *\left(\tilde{V}_{L}^{a}\left(F_{a}\right) B\right)=\tilde{V}_{L}^{a}\left(F_{a}\right) B
$$

for any string field $B$ where $\tilde{V}_{R}^{a}\left(F_{a}\right)=\int_{C_{\text {right }}} \frac{d z}{2 \pi i} F_{a}(z) \tilde{v}^{a}(z)$, we obtain

$$
e^{-\Phi_{0}} * Q_{\mathrm{B}} e^{\Phi_{0}}=\left(e^{\tilde{V}_{L}^{a}\left(F_{a}\right)} Q_{\mathrm{B}} e^{-\tilde{V}_{L}^{a}\left(F_{a}\right)}\right) I=\left(-V_{L}^{a}\left(F_{a}\right)+\frac{1}{8} \Omega^{a b} C_{L}\left(F_{a} F_{b}\right)\right) I,
$$

where

$$
V_{L}^{a}\left(F_{a}\right)=\int_{C_{\text {left }}} \frac{d z}{2 \pi i} F_{a}(z) v^{a}(z), \quad v^{a}(z) \equiv \frac{1}{\sqrt{2}} c J^{a}(z)+\frac{1}{\sqrt{2}} \eta e^{\phi} \psi^{a}(z) .
$$

In the above computation, we have used the relations

$$
\begin{aligned}
& {\left[Q_{\mathrm{B}}, \tilde{v}^{a}(z)\right]=v^{a}(z),} \\
& \tilde{v}^{a}(y) v^{b}(z) \sim \frac{1}{y-z} \frac{-1}{4} \Omega^{a b} c(z), \quad\left[\tilde{V}_{L}^{a}(f), V_{L}^{b}(g)\right]=-\frac{1}{4} \Omega^{a b} C_{L}(f g),
\end{aligned}
$$

which follow from (3.7), (3.9), (3.1) and (3.2). Because both $V_{L}^{a}\left(F_{a}\right)$ and $C_{L}\left(F_{a} F_{b}\right)$ in (3.20) do not include $\xi_{0}$, we conclude that $\Phi_{0}(3.16)$ satisfies the equation of motion:

$$
\eta_{0}\left(e^{-\Phi_{0}} * Q_{\mathrm{B}} e^{\Phi_{0}}\right)=0
$$

By replacing $F_{a}(z)$ with $t F_{a}(z)$, we have $\eta_{0}\left(e^{-t \Phi_{0}} Q_{\mathrm{B}} e^{t \Phi_{0}}\right)=0(0 \leq t \leq 1)$, which implies that the value of the action at this solution is zero: $S\left[\Phi_{0}\right]=0$ as we can easily check from eq. (3.14).

Around the solution $\Phi_{0}$, using (3.20) and (B.19) and noting

$$
A *\left(V_{L}^{a}\left(F_{a}\right) I\right)=-(-1)^{|A|}\left(V_{R}^{a}\left(F_{a}\right) A\right) * I=-(-1)^{|A|} V_{R}^{a}\left(F_{a}\right) A
$$

$\left(V_{R}^{a}(f)=\int_{C_{\text {right }}} \frac{d z}{2 \pi i} F_{a}(z) v^{a}(z)\right)$ for any string field $A$ because $v^{a}(z)$ is a primary field with dimension 0, the new BRS operator $Q_{\mathrm{B}}^{\prime}$ which is a derivation with respect to the star product becomes

$$
\begin{aligned}
& Q_{\mathrm{B}}^{\prime} B \\
& =Q_{\mathrm{B}} B+\left[\left(-V_{L}^{a}\left(F_{a}\right)+\frac{1}{8} \Omega^{a b} C_{L}\left(F_{a} F_{b}\right)\right) I\right] * B-(-1)^{|B|} B *\left[\left(-V_{L}^{a}\left(F_{a}\right)+\frac{1}{8} \Omega^{a b} C_{L}\left(F_{a} F_{b}\right)\right) I\right] \\
& =\left(Q_{\mathrm{B}}-\left(V_{L}^{a}\left(F_{a}\right)+V_{R}^{a}\left(F_{a}\right)\right)+\frac{1}{8} \Omega^{a b}\left(C_{L}\left(F_{a} F_{b}\right)+C_{R}\left(F_{a} F_{b}\right)\right)\right) B
\end{aligned}
$$


on any string field $B$, namely,

$$
\begin{aligned}
& Q_{\mathrm{B}}^{\prime}=Q_{\mathrm{B}}-V^{a}\left(F_{a}\right)+\frac{1}{8} \Omega^{a b} C\left(F_{a} F_{b}\right), \\
& V^{a}\left(F_{a}\right)=\oint \frac{d z}{2 \pi i} F_{a}(z) v^{a}(z), \quad C\left(F_{a} F_{b}\right)=\oint \frac{d z}{2 \pi i} F_{a}(z) F_{b}(z) c(z) .
\end{aligned}
$$

We can directly check $\left\{\eta_{0}, Q_{\mathrm{B}}^{\prime}\right\}=0$ and nilpotency $Q_{\mathrm{B}}^{\prime 2}=0$ noting $\left\{Q_{\mathrm{B}}, v^{a}(z)\right\}=0$ from (3.22) and

$$
v^{a}(y) v^{b}(z) \sim \frac{-\Omega^{a b}}{4(y-z)}\left(c \partial c-\eta \partial \eta e^{2 \phi}\right)(z), \quad\left\{V^{a}\left(F_{a}\right), V^{b}\left(F_{b}\right)\right\}=\frac{-\Omega^{a b}}{4}\left\{Q_{\mathrm{B}}, C\left(F_{a} F_{b}\right)\right\} .
$$

We note that the above BRS operator $Q_{\mathrm{B}}^{\prime}$ is obtained by replacing the matter Virasoro operators $G(z), T(z)$ in (3.15) with $G(z)-\frac{1}{\sqrt{2}} F_{a}(z) \psi^{a}(z), T(z)-\frac{1}{\sqrt{2}} F_{a}(z) J^{a}(z)+\frac{1}{8} \Omega^{a b} F_{a}(z) F_{b}(z)$ respectively. In fact, if we define $G^{\prime}(z)=\sum_{r} G_{r}^{\prime} z^{-r-3 / 2}$ and $T^{\prime}(z)=\sum_{n} L_{n}^{\prime} z^{-n-2}$ as

$$
\begin{aligned}
G_{r}^{\prime} & =G_{r}-\frac{1}{\sqrt{2}} \sum_{k} F_{a, k} \psi_{r-k}^{a}, \\
L_{n}^{\prime} & =L_{n}-\frac{1}{\sqrt{2}} \sum_{k} F_{a, k} J_{n-k}^{a}+\frac{1}{8} \Omega^{a b} \sum_{k} F_{a, n-k} F_{b, k},
\end{aligned}
$$

where $\psi^{a}(z)=\sum_{r} \psi_{r}^{a} z^{-r-1 / 2}, J^{a}(z)=\sum_{n} J_{n}^{a} z^{-n-1}, G(z)=\sum_{r} G_{r} z^{-r-3 / 2}, T(z)=\sum_{n} L_{n} z^{-n-2}$ and $F_{a, n}=\oint \frac{d \sigma}{2 \pi} e^{i(n+1) \sigma} F_{a}\left(e^{i \sigma}\right)$, then, using OPEs among $\left(\psi^{a}, J^{a}, G, T\right)$, we can check that they satisfy the super Virasoro algebra:

$$
\begin{aligned}
& {\left[L_{m}^{\prime}, L_{n}^{\prime}\right]=(m-n) L_{m+n}^{\prime}+\frac{c}{12}\left(m^{3}-m\right) \delta_{m+n, 0}} \\
& \left\{G_{r}^{\prime}, G_{s}^{\prime}\right\}=2 L_{r+s}^{\prime}+\frac{c}{12}\left(4 r^{2}-1\right) \delta_{r+s, 0} \\
& {\left[L_{m}^{\prime}, G_{r}^{\prime}\right]=\left(\frac{m}{2}-r\right) G_{m+r}^{\prime}}
\end{aligned}
$$

with the same central charge as original $G(z), T(z)$ system. Furthermore, let us define $\psi^{\prime a}(z)=$ $\sum_{r} \psi_{r}^{\prime a} z^{-r-1 / 2}$ and $J^{\prime a}(z)=\sum_{n} J_{n}^{\prime a} z^{-n-1}$ by

$$
\psi_{r}^{\prime a}=\sum_{k} M_{b, k}^{a} \psi_{r-k}^{b}, \quad J_{n}^{\prime a}=\sum_{k} M_{b, k}^{a}\left(J_{n-k}^{b}-\frac{1}{2 \sqrt{2}} \Omega^{b c} F_{c, n-k}\right),
$$

where $M_{b, n}^{a}$ is given by a path-ordered form:

$$
\begin{aligned}
M_{b}^{a}(\sigma) & =\sum_{n} M_{b, n}^{a} e^{-i n \sigma}=\left[\mathbf{P} \exp \left(i \int_{0}^{1} d t \sigma A(t \sigma)\right)\right]_{b}^{a} \\
& =\delta_{b}^{a}+\sum_{n=1}^{\infty} i^{n} \sigma^{n} \int_{0}^{1} d t_{1} \int_{0}^{t_{1}} d t_{2} \cdots \int_{0}^{t_{n-1}} d t_{n} A_{c_{n}}^{a}\left(t_{n} \sigma\right) A_{c_{n-1}}^{c_{n}}\left(t_{n-1} \sigma\right) \cdots A_{b}^{c_{2}}\left(t_{1} \sigma\right) \\
A_{b}^{a}(\sigma) & \equiv \frac{1}{\sqrt{2}} f_{b}^{a c} e^{i \sigma} F_{c}\left(e^{i \sigma}\right) .
\end{aligned}
$$


Noting the identities for invariant metric $\Omega^{a b}$ (3.4) and the Jacobi identity for structure constants $f_{c}^{a b}$, we can show following relations:

$$
\begin{array}{ll}
-i \partial_{\sigma} M_{b}^{a}(\sigma)=M_{c}^{a}(\sigma) A_{b}^{c}(\sigma), & -n M_{b, n}^{a}=\frac{1}{\sqrt{2}} \sum_{k} M_{d, k}^{a} f_{b}^{d c} F_{c, n-k}, \\
M_{c}^{a}(\sigma) M_{d}^{b}(\sigma) \Omega^{c d}=\Omega^{a b}, & \sum_{k} M_{c, k}^{a} M_{d, n-k}^{b} \Omega^{c d}=\Omega^{a b} \delta_{n, 0}, \\
M_{d}^{a}(\sigma) M_{e}^{b}(\sigma) f_{c}^{d e}=f_{d}^{a b} M_{c}^{d}(\sigma), & \sum_{k} M_{d, k}^{a} M_{e, n-k}^{b} f_{c}^{d e}=f_{d}^{a b} M_{c, n}^{d},
\end{array}
$$

and we obtain commutation relations:

$$
\begin{aligned}
& \left\{\psi_{r}^{\prime a}, \psi_{s}^{\prime b}\right\}=\frac{1}{2} \Omega^{a b} \delta_{r+s, 0},\left[J_{m}^{\prime a}, J_{n}^{\prime b}\right]=\frac{1}{2} \Omega^{a b} m \delta_{m+n, 0}+f_{c}^{a b} J_{m+n}^{\prime c},\left[J_{n}^{\prime a}, \psi_{r}^{\prime b}\right]=f_{c}^{a b} \psi_{n+r}^{\prime c} \\
& {\left[L_{n}^{\prime}, \psi_{r}^{\prime a}\right]=-\left(\frac{n}{2}+r\right) \psi_{n+r}^{\prime a}, \quad\left[L_{m}^{\prime}, J_{n}^{\prime a}\right]=-n J_{m+n}^{\prime a}} \\
& \left\{G_{r}^{\prime}, \psi_{s}^{\prime a}\right\}=J_{r+s}^{\prime a}, \quad\left[G_{r}^{\prime}, J_{n}^{\prime a}\right]=-n \psi_{n+r}^{\prime a}
\end{aligned}
$$

which are the same form as the original (unprimed) ones.

After all, by re-expanding the action (3.14) around a classical solution $\Phi_{0}\left(\right.$ (3.16) as $e^{\Phi}=$ $e^{\Phi_{0}} e^{\Phi^{\prime}}$, we obtain the action $S^{\prime}\left[\Phi^{\prime}\right]$ with new BRS operator (3.27), which is realized by a replacement $\left(\psi^{a}, J^{a}, G, T\right) \rightarrow\left(\psi^{\prime a}, J^{\prime a}, G^{\prime}, T^{\prime}\right)$ in eqs. (3.30), (3.31) and (3.35) preserving algebra among them. This fact and vanishing vacuum energy: $S\left[\Phi_{0}\right]=0$ suggest that the classical solution $\Phi_{0}$ (3.16) might be a pure gauge solution in terms of superstring field theory. Indeed, the new BRS operator (3.27) can be rewritten as a similarity transform from the original one:

$$
Q_{\mathrm{B}}^{\prime}=e^{\tilde{V}^{a}\left(F_{a}\right)} Q_{\mathrm{B}} e^{-\tilde{V}^{a}\left(F_{a}\right)}, \quad \tilde{V}^{a}\left(F_{a}\right)=\oint \frac{d z}{2 \pi i} F_{a}(z) \tilde{v}^{a}(z)
$$

One might think that the original action $S\left[\Phi^{\prime \prime}\right]$ could be reproduced by a field redefinition such as $\Phi^{\prime \prime}=e^{-\tilde{V}^{a}\left(F_{a}\right)} \Phi^{\prime}=e^{-\tilde{V}_{L}^{a}\left(F_{a}\right) I} * \Phi^{\prime} * e^{\tilde{V}_{L}^{a}\left(F_{a}\right) I}$ in the re-expanded action $S^{\prime}\left[\Phi^{\prime}\right]$. However, it is not so trivial because there is another derivation $\eta_{0}$ in the action and $\left[\eta_{0}, \tilde{V}^{a}\left(F_{a}\right)\right] \neq 0$.

In the following, we demonstrate that if the function $F_{a}(z)$ satisfies a condition, we can explicitly rewrite our solution in a pure gauge form and take an appropriate field redefinition around it, which recovers original action. Let us consider a particular pure gauge form and try to rewrite our solution to it. Noting the commutation relation $\left[Q_{\mathrm{B}}, J^{a}(z)-\partial\left(c \xi e^{-\phi} \psi^{a}\right)(z)\right]=0$, we find an identity:

$$
\begin{aligned}
J^{a}(z) & =\partial\left(c \xi e^{-\phi} \psi^{a}\right)(z)+\left\{Q_{\mathrm{B}}, \Omega^{a}(z)\right\} \\
\Omega^{a}(z) & \equiv \frac{1}{2} c \partial c \xi \partial \xi \partial^{2} \xi e^{-3 \phi} \psi^{a}(z)-c \xi \partial \xi e^{-2 \phi} J^{a}(z)
\end{aligned}
$$


which relates $\tilde{v}^{a}$ (3.18) to the current $J^{a}$ and we have

$$
\begin{aligned}
& \tilde{V}_{L}^{a}\left(\partial g_{a}\right)+J_{L}^{a}\left(g_{a}\right)=\left\{Q_{\mathrm{B}}, \Omega_{L}^{a}\left(g_{a}\right)\right\} \\
& J_{L}^{a}\left(g_{a}\right)=\int_{C_{\text {left }}} \frac{d z}{2 \pi i} \frac{1}{\sqrt{2}} g_{a}(z) J^{a}(z), \quad \Omega_{L}^{a}\left(g_{a}\right)=\int_{C_{\text {left }}} \frac{d z}{2 \pi i} \frac{1}{\sqrt{2}} g_{a}(z) \Omega^{a}(z), \quad g_{a}( \pm i)=0 .
\end{aligned}
$$

The last condition is necessary to remove unwanted boundary contribution of the integration given by $g_{a}( \pm i) \tilde{v}^{a}( \pm i)$. We notice commutation relations

$$
\left[\tilde{V}_{L}^{a}\left(F_{a}\right), \tilde{V}_{L}^{b}\left(G_{b}\right)\right]=0, \quad\left[\tilde{V}_{L}^{a}\left(F_{a}\right), J_{L}^{b}\left(g_{b}\right)\right]=\frac{1}{\sqrt{2}} f_{c}^{a b} \tilde{V}_{L}^{c}\left(F_{a} g_{b}\right),
$$

and a kind of Hausdorff formula:

$$
e^{A} e^{B}=\exp \left(A+\operatorname{ad}_{\frac{A}{2}}\left(1+\operatorname{coth}\left(\operatorname{ad}_{\frac{A}{2}}\right)\right) B+\mathrm{O}\left(B^{2}\right)\right)
$$

where we have denoted $\operatorname{ad}_{X} Y=[X, Y]$ and $\mathrm{O}\left(B^{2}\right)$ is quadratic and higher terms with respect to $B$. By substituting $A=\tilde{V}_{L}^{a}\left(\partial g_{a}\right)+J_{L}^{a}\left(g_{a}\right)$ and $B=-\tilde{V}_{L}^{a}\left(F_{a}\right)$ to the above formula, we obtain

$$
\begin{aligned}
e^{\tilde{V}_{L}^{a}\left(\partial g_{a}\right)+J_{L}^{a}\left(g_{a}\right)} e^{-\tilde{V}_{L}^{a}\left(F_{a}\right)} & =e^{J_{L}^{a}\left(g_{a}\right)+\tilde{V}_{L}^{a}\left(\partial g_{a}-F_{b}\left(\mathcal{M}\left(e^{\mathcal{M}}-1\right)^{-1}\right)_{a}^{b}\right)}, \\
\mathcal{M}_{a}^{b}(z) & =\frac{1}{\sqrt{2}} f_{a}^{b c} g_{c}(z),
\end{aligned}
$$

because $\mathrm{O}\left(B^{2}\right)$ in the exponent vanishes due to (3.49). If the second term in the exponent on the right hand side of the first line vanishes, we can compute as:

$$
e^{Q_{\mathrm{B}} \Omega_{L}^{a}\left(g_{a}\right) I} e^{-\tilde{V}_{L}^{a}\left(F_{a}\right) I}=e^{\left(\tilde{V}_{L}^{a}\left(\partial g_{a}\right)+J_{L}^{a}\left(g_{a}\right)\right)} e^{-\tilde{V}_{L}^{a}\left(F_{a}\right)} I=e^{J_{L}^{a}\left(g_{a}\right)} I=e^{\eta_{0} \xi_{0} J_{L}^{a}\left(g_{a}\right) I},
$$

where we should impose $g_{a}(-1 / z)=g_{a}(z)$ to guarantee a relation such as eq. (2.20). The above calculation means that, by solving a differential equation with respect to $g_{a}(z)$ :

$$
\begin{aligned}
& F_{b}(z)=\partial g_{a}(z)\left(\left(e^{\mathcal{M}}-1\right) \mathcal{M}^{-1}\right)_{b}^{a}(z) \\
& g_{a}(-1 / z)=g_{a}(z), \quad g_{a}( \pm i)=0
\end{aligned}
$$

for a given $F_{a}(z)$ which specifies the classical solution $\Phi_{0}$ (3.16), we obtain the form of gauge transformation from the trivial solution $\Phi=0$ in superstring field theory:

$$
e^{\Phi_{0}}=e^{Q_{\mathrm{B}}\left(-\Omega_{L}^{a}\left(g_{a}\right)\right) I} e^{\eta_{0}\left(\xi_{0} J_{L}^{a}\left(g_{a}\right)\right) I}
$$

Note that the equation (3.54) is consistent with the conditions $F_{a}(-1 / z)=z^{2} F_{a}(z)$ and $g_{a}(-1 / z)=g_{a}(z)$. For an abelian $\mathcal{G}$, where $\mathcal{M}_{a}^{b}(z)=0$, it becomes a simple form $\partial g_{a}(z)=$ 
$F_{a}(z)$ because of $\left(\left(e^{\mathcal{M}}-1\right) \mathcal{M}^{-1}\right)_{b}^{a}(z)=\delta_{b}^{a}+\mathrm{O}(\mathcal{M})$. Similarly, we can rewrite the new BRS operator (3.27) as

$$
Q_{\mathrm{B}}^{\prime}=e^{-\Lambda} Q_{\mathrm{B}} e^{\Lambda}, \quad \Lambda=-\oint \frac{d z}{2 \pi i} \frac{1}{\sqrt{2}} g_{a}(z) J^{a}(z)
$$

using the above $g_{a}$. Thanks to $\left[\eta_{0}, \Lambda\right]=0$, we can recover the original action by taking a field redefinition $\Phi^{\prime \prime}=e^{\Lambda} \Phi^{\prime}=e^{\frac{1}{2} \lambda_{a} \oint \frac{d z}{2 \pi} J^{a}(z)+\cdots} \Phi^{\prime}$, which corresponds to a marginal deformation by the current $J^{a}$ [46]. Its deformation parameter $\lambda_{a}=\sqrt{2} i \int_{-\pi}^{\pi} \frac{d \sigma}{2 \pi} g_{a}\left(e^{i \sigma}\right)$ is related to the function $F_{a}$ in our classical solution by eq. (3.54). However, a solution to the differential equation (3.54) which satisfies the conditions (3.55) does not necessarily exist. In fact, eq. (3.54) can be rewritten as,

$$
\frac{1}{\sqrt{2}} F_{b}(z) T^{b}=e^{-\frac{1}{\sqrt{2}} g_{a}(z) T^{a}} \partial\left(e^{\frac{1}{\sqrt{2}} g_{a}(z) T^{a}}\right),
$$

where $T^{a} \mathrm{~S}$ are generator matrices of $\mathcal{G}$ such as $\left[T^{a}, T^{b}\right]=f^{a b}{ }_{c} T^{c}$ and use has been made of $\left(e^{\mathcal{M}}-1\right) \mathcal{M}^{-1}=\int_{0}^{1} d t e^{t \mathcal{M}}$ and $\delta\left(e^{X}\right)=\int_{0}^{1} d t e^{(1-t) X} \delta X e^{t X}$. This equation can be solved by path-ordered form:

$$
e^{\frac{1}{\sqrt{2}} g_{a}\left(e^{i \sigma}\right) T^{a}}=\mathbf{P} e^{\frac{i}{\sqrt{2}} \int_{0}^{1} d t\left(\sigma-\operatorname{sgn}(\sigma) \frac{\pi}{2}\right) e^{i t \sigma+i \operatorname{sgn}(\sigma) \frac{\pi}{2}(1-t)} F_{a}\left(e^{i t \sigma+i \operatorname{sgn}(\sigma) \frac{\pi}{2}(1-t)}\right) T^{a}},
$$

where the path ordering denoted by $\mathbf{P}$ is taken as (3.36) (i.e., we put a matrix associated with larger $t$ to the right) and $\operatorname{sgn}(\sigma)=+1(-1)$ for $\sigma>0(\sigma<0)$ is used. Here we have respected the second condition in (3.55): $g_{a}( \pm i)=0$ and solved separately on upper and lower half circle by taking the phase of $z=e^{i \sigma}$ as $-\pi \leq \sigma \leq \pi$ on the unit circle. The property of the function $F_{a}(z)$ in our solution $\Phi_{0}$ (3.16)): $F_{a}(-1 / z)=z^{2} F_{a}(z)$ implies $e^{i(\operatorname{sgn}(\sigma) \pi-\sigma)} F_{a}\left(e^{i(\operatorname{sgn}(\sigma) \pi-\sigma)}\right)=$ $-e^{i \sigma} F_{a}\left(e^{i \sigma}\right)$ and then the above solution satisfies the first condition in (3.55) $): g_{a}\left(e^{i(\operatorname{sgn}(\sigma) \pi-\sigma)}\right)=$ $g_{a}\left(e^{i \sigma}\right)$. In order to guarantee the continuity of $g_{a}\left(e^{i \sigma}\right)$ at $\sigma=0$, which is needed for (3.47), there is a consistency condition for $F_{a}(z)$ :

$$
\mathbf{P} e^{\frac{-i \pi}{2 \sqrt{2}} \int_{0}^{1} d t e^{i \frac{\pi}{2}(1-t)} F_{a}\left(e^{i \frac{\pi}{2}(1-t)}\right) T^{a}}=\mathbf{P} e^{\frac{i \pi}{2 \sqrt{2}} \int_{0}^{1} d t e^{-i \frac{\pi}{2}(1-t)} F_{a}\left(e^{-i \frac{\pi}{2}(1-t)}\right) T^{a}},
$$

which is reduced to

$$
\int_{C_{\text {left }}} d z F_{a}(z)=0
$$

in the case of $\mathcal{G}$ : abelian. Namely, if $F_{a}(z)$ satisfies the condition (3.601) (or (3.61) for abelian $\mathcal{G})$, the solution (3.16) is rewritten in a pure gauge form (3.56) and induces a field redefinition generated by $\Lambda$ in (3.57). Conversely, in the case that $F_{a}(z)$ breaks the condition (3.60), we 
cannot rewrite as (3.56) and we should know further informations about the supercurrent and its representation by specifying a model which realizes $\mathbf{J}^{a}(z, \theta)$ in order to find explicit relations between our solution and marginal deformation.

As an example of $\mathcal{G}=u(1)^{10}$, we take an ordinary flat background which is described by a supercurrent $\mathbf{J}^{\mu}(z, \theta)=\psi^{\mu}(z)+\theta \frac{i}{\sqrt{2 \alpha^{\prime}}} \partial X^{\mu}(z)$. In this case, we can identify various quantities as follows:

$$
\begin{aligned}
& T(z)=-\frac{1}{4 \alpha^{\prime}} \partial X^{\mu} \partial X_{\mu}(z)-\frac{1}{2} \psi^{\mu} \partial \psi_{\mu}(z), \quad G(z)=\frac{i}{\sqrt{2 \alpha^{\prime}}} \partial X_{\mu} \psi^{\mu}(z) \\
& \Omega^{\mu \nu}=2 \eta^{\mu \nu}, \quad \Omega_{\mu \nu}=\frac{1}{2} \eta_{\mu \nu}, \quad f_{\rho}^{\mu \nu}=0, \quad c=\frac{3}{2} \operatorname{dim}\left(u(1)^{10}\right)=15
\end{aligned}
$$

By taking functions $F_{\mu}(z)$ for a solution $\Phi_{0}$ (3.16) as $F_{\mu}(z)=\delta_{\mu, 9} F(z)$ such as $F(-1 / z)=$ $z^{2} F(z)$, we reproduce the solution in the previous section, which has turned out to correspond to the Wilson line. From the condition (3.61), non-vanishing Wilson line $f=\int_{C_{\text {left }}} \frac{d z}{2 \pi i} F(z) \neq 0$ implies non-existence of a function $g_{\mu}(z)$ which specifies globally defined gauge parameter of the form (3.56) and a field redefinition associated with (3.57). Instead, we have found other (local) expressions (2.60) and (2.58) using the integration of the current $J^{\mu}(z)$.

We comment on the analogy with arguments in the Witten's bosonic string field theory (see appendix (D). In both cases, we can construct a class of classical solutions (3.16), (D.11) based on (super-)currents, which have vanishing vacuum energy. The actions around the solution are also obtained by appropriate operator mappings (3.35), (D.15) which preserve current algebra in both cases. This fact suggests that the theory around the solutions is essentially the same as the original one and they might be gauge equivalent. Indeed, we can represent the solutions as a pure gauge form in both supersymmetric and bosonic string field theory if there exists a solution $g_{a}(z)$ to the differential equation (3.54), which is the same form as (D.21). In both cases, the change of BRS operator around the solution turns out to be absorbed by a field redefinition using $g_{a}(z)$. As was shown in the previous section for supersymmetric case and in ref. [21] for bosonic case, we can obtain nontrivial solutions in a global sense by considering a compactified background with the non-vanishing Wilson lines $\int_{C_{\text {left }}} d z F_{\mu}(z) \neq 0$. In general case, there is a possibility that our classical solutions become nontrivial if $F_{a}(z)$ breaks the condition (3.60), although we cannot prove their non-triviality with respect to gauge transformation of string field theory at this stage because we have only investigated a particular pure gauge form: (3.56) or (D.20). We speculate that they will be rewritten as a locally pure gauge form using a kind of integration of the current in each model in the case that $g_{a}(z)$ does not exist. 


\section{Discussions}

We constructed a class of analytic classical solutions in open superstring field theory, which is related to marginal deformations in conformal field theory. We showed that the resulting solutions can be represented using a well-defined Fock space expression, and the vacuum energy vanishes due to the ghost number non-conservation in the large Hilbert space. For the solution corresponding to background Wilson lines, we observe that the solution can be written as a locally pure gauge form, and the action expanded around the solution can be transformed locally back to the original action by a string field redefinition. The analytic classical solution enabled us to investigate gauge structure in the string field theory. We found that the half integration mode of the function in the solution is unchanged under the "global transformation", but other modes can be gauged away. Space-time supersymmetry is realized on-shell in the theory and the solution is a supersymmetric solution. We note that the classical solutions in the present paper can be easily extended to that of the theory given in ref. [8] including the $\mathrm{GSO}(-)$ sector

We have extensively used formal properties of the identity string field to construct our solution and to investigate its structure. In general, the identity string field requires careful handling to evaluate some quantities of the form $\left\langle\left\langle\left(\mathcal{O}_{1} I\right)\left(\mathcal{O}_{2} I\right)\right\rangle\right.$. Actually, in terms of oscillator representation, we encounter divergence from contractions of nonzero modes in computing $\langle I|\cdots| I\rangle$ as in the case of bosonic string field theory. Consequently, it is necessary to define appropriate regularization of the identity string field, which is not yet known, in both super and bosonic string field theory. However, we evaluated the vacuum energy at our solution $\Phi_{0}$ using the zero-mode saturation rule for the $\xi \eta$ ghost system in the large Hilbert space. We hope to obtain information about a consistent regularization by comparing these calculations. The situation is different in bosonic string field theory, where we gave a formal proof (D.18) to show that the vacuum energy at the solution corresponding to the marginal deformation vanishes as in refs. [22, 47]. It is preferable to prove it more directly.

We briefly comment on the modified version of cubic superstring field theory [35, 36]. Setting the Ramond field to zero, the equation of motion is given by $Y_{-2}\left(Q_{\mathrm{B}} A+A * A\right)=0$, where $Y_{-2}$ is the picture changing operator with picture number $(-2)$. We note that a string field $A_{0}=e^{-\Phi_{0}} Q_{\mathrm{B}} e^{\Phi_{0}}$, in which $\Phi_{0}$ is our solution in the Berkovits' theory, is a solution to the equation of motion. In fact, $Q_{\mathrm{B}} A_{0}+A_{0} * A_{0}=0$ holds and $A_{0}$ itself has ghost number 1 and picture number 0 and is Grassmann odd in the small Hilbert space. Therefore, we find that $A_{0}$ is pure gauge with the gauge parameter $J_{L}^{a}\left(g_{a}\right) I$ if $F_{a}(z)$ in $\Phi_{0}$ satisfies the condition (3.60) . 
We notice that $\Phi_{0}$ itself cannot be a gauge parameter in spite of the form of $A_{0}$ because $\Phi_{0}$ is not in the small Hilbert space : $\eta_{0} \Phi_{0} \neq 0$.

For the Wilson line solution, the half integration of the function is unchanged under the global transformation. This is an obvious result because the half integration mode as the Wilson line should be a physical observable. However, we confirmed the invariance of the half integration mode merely for a part of the gauge symmetry, and it is difficult to prove the invariance for the whole gauge symmetry. More precisely, we have to relate the solution to a general gauge invariant quantity in string field theory. Although a gauge invariant quantity plays important roles in field theories, we have not yet understood completely how it can be constructed in string field theory. As gauge invariants, we know the action and some operators only [48, 49]. It is natural to ask how the Wilson loop operator is generalized in string field theory. This is an important open question.

In the theory expanded around the classical solution, the background of the theory can be changed from the unexpanded theory. If we choose $s u(2)$ currents, the solution corresponds to the tachyon lump solution [21, 23] (see also appendix D). In the background, the boundary condition of a string coordinate is changed from the Neumann one to the Dirichlet one [1]. In string field theory, we found this phenomenon indirectly by studying a gauge invariant operator 23]. On the other hand, in ref. [47], it was proposed that string coordinates $X^{\mu}(\sigma)$ and its conjugate momenta $P_{\mu}(\sigma)$ are universal objects in string field theory, and the various backgrounds correspond to inequivalent representations of their canonical algebra. In the present case, it seems that the tachyon lump solution changes representation of universal coordinates, namely their boundary conditions. This subject was studied from the viewpoint of vacuum string field theory [50].

It is an important problem to construct an analytic solution representing tachyon condensation in the superstring field theory. In order to investigate this subject, we have to introduce Chan-Paton matrices to include both $\mathrm{GSO}(+)$ and $\mathrm{GSO}(-)$ sectors. Such a formulation were developed in ref. [8]. We hope to obtain an analytic expression of the tachyon vacuum by an analogous construction to the bosonic case (i.e., a class of scalar solutions in refs. [22, 37]).

\section{Acknowledgements}

The authors would like to thank Yuji Igarashi and Katsumi Itoh for useful discussions. I. K. wishes to express his gratitude to Kazuki Ohmori for valuable comments. 


\section{A Oscillator expression of the identity string field}

We use the identity string field in constructing exact solutions in the framework of Berkovits' open superstring field theory. The action is described in terms of the large Hilbert space [51] in the NSR formalism. We shall give an explicit oscillator representation of the identity string field $|I\rangle$ in terms of modes of $X^{\mu}, \psi^{\mu}, b, c, \phi, \xi, \eta$ in the NS sector. In this paper, we formally regard the identity string field $I$ as the identity element with respect to the Witten $*$ product: $A * I=I * A=A$, which should be proved by LPP's definition of string vertices [38] and generalized gluing and resmoothing theorem at the critical dimension $d=10$ [39, 52, 53]. Therefore, we define $\langle I|$ as a 1-string LPP vertex using conformal mapping: $h_{I}(z)=2 z /\left(1-z^{2}\right)$ [54] and CFT correlator in the large Hilbert space denoted by $\langle\langle\cdots\rangle\rangle$ which is evaluated on the upper half plane: ${ }^{20}\langle I \mid A\rangle=\left\langle\left\langle h_{I}\left[\mathcal{O}_{A}(0)\right]\right\rangle\right.$, where $\left.\mid A\right\rangle=\mathcal{O}_{A}(0)|0\rangle$ is an arbitrary state. In ref. [38, the integral expression for Neumann coefficients in $X^{\mu}, b, c$ sector is given with this definition and the oscillator expression of the identity string filed is obtained by applying the conformal map $h_{I}(z)$, which is consistent with that in [42, 55]. The result is

$$
\begin{aligned}
|I\rangle_{b} & =e^{E_{X b c}}\left|p^{\mu}=0\right\rangle \\
E_{X b c} & =\sum_{n \geq 1} \frac{-(-1)^{n}}{2 n} \alpha_{-n}^{\mu} \alpha_{-n \mu}+\sum_{n \geq 2}(-1)^{n} c_{-n} b_{-n}-\sum_{k \geq 1}(-1)^{k}\left(2 c_{0} b_{-2 k}+\left(c_{1}-c_{-1}\right) b_{-2 k-1}\right),
\end{aligned}
$$

which is the same as the identity string field in the Witten's bosonic open string field theory if $\mu$ runs over $0,1, \cdots, 25$.

In the same way, we can calculate the Neumann coefficients in the matter fermion sector $\left(\psi^{\mu}\right)$ as:

$$
\begin{aligned}
I_{r s} & =-I_{s r}=\oint_{0} \frac{d y}{2 \pi i} y^{-r-\frac{1}{2}} \oint_{0} \frac{d z}{2 \pi i} z^{-s-\frac{1}{2}} \frac{\left(h_{I}^{\prime}(y)\right)^{\frac{1}{2}}\left(h_{I}^{\prime}(z)\right)^{\frac{1}{2}}}{h_{I}(y)-h_{I}(z)} \\
& =\left\{\begin{array}{cc}
-\frac{r(2 s-1)}{r^{2}-s^{2}}\left(\frac{-1}{4}\right)^{\frac{r+s}{2}} \frac{\left(r-\frac{1}{2}\right) !\left(s-\frac{3}{2}\right) !}{\left[\left(\frac{1}{2}\left(r-\frac{1}{2}\right)\right) !\left(\frac{1}{2}\left(s-\frac{3}{2}\right)\right) !\right]^{2}} & \left(r-\frac{1}{2}: \text { even } ; s-\frac{1}{2}: \text { odd }\right) \\
-\frac{s(2 r-1)}{r^{2}-s^{2}}\left(\frac{-1}{4}\right)^{\frac{r+s}{2}} \frac{\left(r-\frac{3}{2}\right) !\left(s-\frac{1}{2}\right) !}{\left[\left(\frac{1}{2}\left(r-\frac{3}{2}\right)\right) !\left(\frac{1}{2}\left(s-\frac{1}{2}\right)\right) !\right]^{2}} & \left(r-\frac{1}{2}: \text { odd } ; s-\frac{1}{2}:\right. \text { even) } \\
0 & \text { (otherwise) }
\end{array}\right.
\end{aligned}
$$

where we have used the expansion:

$$
\sum_{r, s \geq \frac{1}{2}}\left(r^{2}-s^{2}\right) I_{r s} y^{r-\frac{1}{2}} z^{s-\frac{1}{2}}=\left(y \partial_{y}-z \partial_{z}\right)\left(y \partial_{y}+z \partial_{z}+1\right)\left(\frac{\left(h_{I}^{\prime}(y)\right)^{\frac{1}{2}}\left(h_{I}^{\prime}(z)\right)^{\frac{1}{2}}}{h_{I}(y)-h_{I}(z)}-\frac{1}{y-z}\right)
$$

\footnotetext{
${ }^{20}$ In this paper, we implicitly use the doubling trick: a holomorphic field $\sigma(z)$ and antiholomorphic one $\tilde{\sigma}(\bar{z})$ in the upper half plane are combined into a holomorphic field defined in the whole complex plane with a boundary condition $\sigma(z)=\tilde{\sigma}(\bar{z})$ on the real axis: $\operatorname{Im} z=0$.
} 


$$
=\frac{y\left(1-z^{2}\right)+z\left(1-y^{2}\right)}{\left(1+y^{2}\right)^{\frac{3}{2}}\left(1+z^{2}\right)^{\frac{3}{2}}}=\sum_{k, l=0}^{\infty}(2 k+1)(4 l+1)\left(\begin{array}{c}
\frac{-1}{2} \\
k
\end{array}\right)\left(\begin{array}{c}
\frac{-1}{2} \\
l
\end{array}\right)\left(y^{2 k+1} z^{2 l}+y^{2 l} z^{2 k+1}\right) .
$$

$\left(\begin{array}{l}a \\ b\end{array}\right)=\frac{\Gamma(a+1)}{\Gamma(b+1) \Gamma(a-b+1)}$ is the binomial coefficient. This formula for the coefficients $I_{r s}$ is consistent with that in [56].

As for the $\phi$ sector, the formula for the Neumann coefficients is slightly different from that of $X^{\mu}$ because of the background charge $Q=2$ [39]. One can compute explicitly by substituting $h_{I}(z)$ into the integrand:

$$
\begin{aligned}
\mathcal{N}_{m n} & =\frac{1}{m n} \oint_{0} \frac{d y}{2 \pi i} y^{-m} \oint_{0} \frac{d z}{2 \pi i} z^{-n} \frac{h_{I}^{\prime}(y) h_{I}^{\prime}(z)}{\left(h_{I}(y)-h_{I}(z)\right)^{2}}=-\frac{(-1)^{m}}{m} \delta_{m, n}, \quad(m, n \geq 1), \\
\mathcal{N}_{0 n} & =-\frac{1}{2 n} \oint_{0} \frac{d w}{2 \pi i} w^{-n} \partial_{w} \log \left(\left.\partial_{y} \partial_{w} \log \left(h_{I}(y)-h_{I}(w)\right)\right|_{y=0}\right)
\end{aligned}
$$

In the $\xi \eta$ sector, the Neumann coefficients for the identity string field are computed as ${ }^{21}$

$$
\begin{aligned}
& N_{m n}=\oint_{0} \frac{d y}{2 \pi i} y^{-m-1} \oint_{0} \frac{d z}{2 \pi i} z^{-n} \frac{-h_{I}^{\prime}(z)}{h_{I}(y)-h_{I}(z)}=-(-1)^{m} \delta_{m, n}, \quad m, n \geq 1, \\
& N_{0 n}=\oint_{0} \frac{d y}{2 \pi i} y^{-1} \oint_{0} \frac{d z}{2 \pi i} z^{-n} \frac{-h_{I}^{\prime}(z)}{h_{I}(y)-h_{I}(z)}=\left\{\begin{array}{cc}
2 & (n: \text { even }) \\
0 & (n: \text { odd })
\end{array}, \quad n \geq 1,\right.
\end{aligned}
$$

where use has been made of the expansion

$$
\frac{h_{I}^{\prime}(z)}{h_{I}(y)-h_{I}(z)}-\frac{1}{y-z}=-\frac{y\left(1+z^{2}\right)+2 z}{\left(1-z^{2}\right)(1+y z)}=-\sum_{k=0}^{\infty}\left(2 z^{2 k+1}+(-1)^{k} y^{k+1} z^{k}\right) .(
$$

After all, the identity string field of the fermionic sector $\left(\psi^{\mu}, \phi, \xi, \eta\right)$ is given by

$$
\begin{aligned}
|I\rangle_{f} & =e^{E_{\psi \phi \xi \eta}}|q=0\rangle \\
E_{\psi \phi \xi \eta} & =\sum_{r, s \geq 1 / 2} \frac{I_{r s}}{2} \psi_{-r}^{\mu} \psi_{-s \mu}+\sum_{n \geq 1} \frac{(-1)^{n}}{2 n}\left(j_{-n}\right)^{2}-\sum_{k \geq 1} \frac{(-1)^{k}}{k} j_{-2 k}+\sum_{n \geq 1}(-1)^{n} \eta_{-n} \xi_{-n},
\end{aligned}
$$

\footnotetext{
${ }^{21}$ In general, $N$-string vertex is given by

$$
\left\langle V_{N}\right|=\prod_{r} r\left(0 \mid \xi_{0}^{r} e^{\sum_{r, s=1}^{N} \sum_{n \geq 0, m \geq 1} \eta_{n}^{r} N_{n m}^{r s} \xi_{m}^{s}}\left(\eta_{0}^{1}+\cdots+\eta_{0}^{N}\right),\right.
$$
}

in this sector, which is obtained using the method in 38]. 
where the oscillators are given by

$$
\begin{aligned}
& \psi^{\mu}(z)=\sum_{r} \psi_{r}^{\mu} z^{-r-1 / 2}, \quad\left\{\psi_{r}^{\mu}, \psi_{s}^{\nu}\right\}=\eta^{\mu \nu} \delta_{r+s, 0} \\
& \phi(z)=\hat{\phi}_{0}-j_{0} \log z+\sum_{n \neq 0} \frac{1}{n} j_{n} z^{-n}, \quad\left[j_{0}, \hat{\phi}_{0}\right]=1, \quad\left[j_{m}, j_{n}\right]=-m \delta_{m+n, 0}, \\
& \xi(z)=\sum_{n} \xi_{n} z^{-n}, \quad \eta(z)=\sum_{n} \eta_{n} z^{-n-1}, \quad\left\{\xi_{m}, \eta_{n}\right\}=\delta_{m+n, 0}
\end{aligned}
$$

and the vacuum with $\phi$-charge $q$ is defined by $|q\rangle=e^{q \phi}(0)|0\rangle=e^{q \hat{\phi}_{0}}|0\rangle$.

Combining (A.1) and (A.11), the identity string field in open superstring field theory in the large Hilbert space is obtained:

$$
|I\rangle=|I\rangle_{b} \otimes|I\rangle_{f}
$$

The index $\mu$ in the exponent of $|I\rangle_{b}$ runs over $0,1, \cdots, 9$ and $\left|p^{\mu}=0\right\rangle \otimes|q=0\rangle$ is the conformal vacuum. $|I\rangle$ is Grassmann even and has both ghost and picture number 0. BRS invariance $Q_{\mathrm{B}}|I\rangle=0$ follows from the construction of LPP vertex and $\eta_{0}|I\rangle=0$ can be checked directly. We can easily derive the following connection conditions of each oscillators on the identity string field $|I\rangle$ using the above explicit expression: ${ }^{22}$

$$
\begin{aligned}
& \left(\alpha_{n}^{\mu}+(-1)^{n} \alpha_{-n}^{\mu}\right)|I\rangle=0, \quad\left(b_{n}-(-1)^{n} b_{-n}\right)|I\rangle=0, \\
& \left(c_{2 k}+c_{-2 k}-(-1)^{k} 2 c_{0}\right)|I\rangle=0, \quad\left(c_{2 k+1}-c_{-(2 k+1)}-(-1)^{k}\left(c_{1}-c_{-1}\right)\right)|I\rangle=0, \\
& \left(\psi_{r}^{\mu}-\sum_{s \geq 1 / 2} I_{r s} \psi_{-s}^{\mu}\right)|I\rangle=0, \quad\left(\xi_{n}-(-1)^{n} \xi_{-n}\right)|I\rangle=0, \quad\left(\eta_{n}+(-1)^{n} \eta_{-n}\right)|I\rangle=0, \\
& \left(j_{2 k}+j_{-2 k}-(-1)^{k} 2\right)|I\rangle=0, \quad\left(j_{2 k-1}-j_{-(2 k-1)}\right)|I\rangle=0, \quad(k \geq 1) ; \quad j_{0}|I\rangle=0 .
\end{aligned}
$$

The identity string field $|I\rangle$ satisfies the reality condition: $(|I\rangle)^{\dagger}=\mathrm{bpz}(|I\rangle)$, where the BPZ conjugation is given by $\operatorname{bpz}\left(\sigma_{n}\right)=(-1)^{-n+h} \sigma_{-n}$ for oscillators of a primary field $\sigma(z)$ with conformal dimension $h$ and $\mathrm{bpz}\left(\left|p^{\mu} ; q\right\rangle\right)=\left(\left|-p^{\mu} ; q\right\rangle\right)^{\dagger}$ for zero mode part and use has been made of $(-1)^{r+s} I_{r s}=I_{r s}$. We note that the identity string field $|I\rangle$ can be rewritten as

$$
\begin{aligned}
|I\rangle= & \frac{(2 i)^{\frac{1}{4}}}{4 i} b(i \pi / 2) b(-i \pi / 2): e^{\frac{1}{2} \phi(i \pi / 2)}:: e^{\frac{1}{2} \phi(-i \pi / 2)}: e^{E^{\prime}} c_{0} c_{1}\left|p^{\mu}=0 ; q=-1\right\rangle, \\
E^{\prime}= & -\frac{1}{2} \sum_{n \geq 1} \frac{(-1)^{n}}{n} \alpha_{-n}^{\mu} \alpha_{-n \mu}+\sum_{n \geq 1}(-1)^{n} c_{-n} b_{-n} \\
& +\frac{1}{2} \sum_{r, s \geq 1 / 2} I_{r s} \psi_{-r}^{\mu} \psi_{-s \mu}+\frac{1}{2} \sum_{n \geq 1} \frac{(-1)^{n}}{n} j_{-n} j_{-n}+\sum_{n \geq 1}(-1)^{n} \eta_{-n} \xi_{-n},
\end{aligned}
$$

\footnotetext{
${ }^{22}$ For a derivation using CFT, see ref. [57].
} 
where we denoted

$$
\begin{aligned}
& b(i \sigma)=\sum_{n} b_{n} e^{-i n \sigma}, \\
& : e^{q \phi(i \sigma)}:=e^{-\frac{1}{2} q(q+2) i \sigma} e^{-q \sum_{n \geq 1} \frac{1}{n} j_{-n} e^{i n \sigma}} e^{q \hat{\phi}_{0}} e^{-i q \sigma j_{0}} e^{q \sum_{n \geq 1} \frac{1}{n} j_{n} e^{-i n \sigma}} .
\end{aligned}
$$

The extra factor $e^{-\frac{1}{2} q(q+2) i \sigma}$ in the normal order form comes from the conformal factor under the map $z=e^{\rho}$.

\section{B Action around a classical solution}

The Berkovits' action for open superstring field theory in the NS sector is given by

$$
S[\Phi]=-\frac{1}{2 g^{2}}\left\langle\left\langle\bar{A}_{\eta_{0}} \bar{A}_{Q}\right\rangle\right\rangle-\frac{1}{2 g^{2}} \int_{0}^{1} d t\left\langle\left\langle A_{t}\left\{A_{Q}, A_{\eta_{0}}\right\}\right\rangle\right\rangle,
$$

where $A_{\eta_{0}}, A_{Q}$ and $A_{t}$ are defined by string field $\Phi(t)$ parametrized by $t$ with boundary value $\Phi(1)=\Phi, \Phi(0)=0$ :

$$
A_{\eta_{0}}=e^{-\Phi(t)}\left(\eta_{0} e^{\Phi(t)}\right), \quad A_{Q}=e^{-\Phi(t)}\left(Q e^{\Phi(t)}\right), \quad A_{t}=e^{-\Phi(t)}\left(\partial_{t} e^{\Phi(t)}\right)
$$

and $\bar{A}_{\eta_{0}}=\left.A_{\eta_{0}}\right|_{t=1}, \bar{A}_{Q}=\left.A_{Q}\right|_{t=1}$. We usually take $\Phi(t)=t \Phi$ although the action $S[\Phi]$ itself does not depend on this parameterization. We often denote $\{A, B\}=A B+B A$ and $[A, B]=A B-B A$ and omit the symbol for the star product among string fields. We note that $\eta_{0}, Q$ and $\partial_{t}$ are derivations with respect to the star product:

$$
\begin{aligned}
\eta_{0}(A * B) & =\left(\eta_{0} A\right) * B+(-1)^{|A|} A *\left(\eta_{0} B\right), \\
Q(A * B) & =(Q A) * B+(-1)^{|A|} A *(Q B), \\
\partial_{t}(A * B) & =\left(\partial_{t} A\right) * B+A *\left(\partial_{t} B\right),
\end{aligned}
$$

where $(-1)^{|A|}$ is $+1(-1)$ when $A$ is Grassmann even (odd) and have nilpotency $\eta_{0}^{2}=Q^{2}=0$ and (anti-)commutativity: $\left\{\eta_{0}, Q\right\}=0,\left[\partial_{t}, \eta_{0}\right]=\left[\partial_{t}, Q\right]=0$. The above WZW type action (B.1) can be rewritten in a rather simple form [34]:

$$
S[\Phi]=-\frac{1}{g^{2}} \int_{0}^{1} d t\left\langle\left\langle\left(\eta_{0} A_{t}\right) A_{Q}\right\rangle\right\rangle .
$$

Let us consider re-expansion of this action around $\Phi^{(0)}$ with respect to $\Phi^{\prime}$ in the sense $e^{\Phi(t)}=$ $e^{\Phi^{(0)}(t)} e^{\Phi^{\prime}(t)}$. The integrand of (B.6) can be rewritten as:

$\left\langle\left\langle\left(\eta_{0} A_{t}\right) A_{Q}\right\rangle\right\rangle=\left\langle\left\langle\left(e^{-\Phi^{\prime}(t)}\left(\eta_{0} A_{t}^{(0)}\right) e^{\Phi^{\prime}(t)}+\eta_{0} A_{t}^{\prime}+\left(\eta_{0} e^{-\Phi^{\prime}(t)}\right) A_{t}^{(0)} e^{\Phi^{\prime}(t)}+e^{-\Phi^{\prime}(t)} A_{t}^{(0)}\left(\eta_{0} e^{\Phi^{\prime}(t)}\right)\right)\right.\right.$ 


$$
\begin{aligned}
& \left.\left.\times\left(e^{-\Phi^{\prime}(t)} A_{Q}^{(0)} e^{\Phi^{\prime}(t)}+A_{Q}^{\prime}\right)\right\rangle\right\rangle \\
= & \left\langle\left\langle\left(\eta_{0} A_{t}^{(0)}\right) A_{Q}^{(0)}\right\rangle\right\rangle+\left\langle\left\langle\left(\eta_{0} A_{t}^{\prime}\right) A_{Q}^{\prime}\right\rangle\right\rangle \\
& +\left\langle\left\langle\left(\eta_{0} A_{t}^{\prime}\right) e^{-\Phi^{\prime}(t)} A_{Q}^{(0)} e^{\Phi^{\prime}(t)}-e^{\Phi^{\prime}(t)}\left(\eta_{0} e^{-\Phi^{\prime}(t)}\right) \partial_{t} A_{Q}^{(0)}\right\rangle\right\rangle \\
& +\left\langle\left\langle\left(\eta_{0} A_{t}^{(0)}\right) e^{\Phi^{\prime}(t)} A_{Q}^{\prime} e^{-\Phi^{\prime}(t)}+\left(\eta_{0} e^{-\Phi^{\prime}(t)}\right) A_{t}^{(0)} e^{\Phi^{\prime}(t)} A_{Q}^{\prime}+e^{-\Phi^{\prime}(t)} A_{t}^{(0)}\left(\eta_{0} e^{\Phi^{\prime}(t)}\right) A_{Q}^{\prime}\right\rangle\right\rangle \\
& +\left\langle\left\langle e^{-\Phi^{\prime}(t)}\left(\eta_{0} e^{\Phi^{\prime}(t)}\right)\left(Q A_{t}^{(0)}\right)\right\rangle\right\rangle,
\end{aligned}
$$

where we denote $A_{t}^{(0)}=e^{-\Phi^{(0)}(t)}\left(\partial_{t} e^{\Phi^{(0)}(t)}\right), A_{Q}^{(0)}=e^{-\Phi^{(0)}(t)}\left(Q e^{\Phi^{(0)}(t)}\right), A_{t}^{\prime}=e^{-\Phi^{\prime}(t)}\left(\partial_{t} e^{\Phi^{\prime}(t)}\right)$, and $A_{Q}^{\prime}=e^{-\Phi^{\prime}(t)}\left(Q e^{\Phi^{\prime}(t)}\right)$ and use has been made of cyclic property:

$$
\begin{aligned}
& \left\langle\left\langle A_{1} \cdots A_{n-1} \Phi\right\rangle\right\rangle=\left\langle\left\langle\Phi A_{1} \cdots A_{n-1}\right\rangle\right\rangle, \\
& \left\langle\left\langle A_{1} \cdots A_{n-1}(Q \Phi)\right\rangle\right\rangle=-\left\langle\left\langle(Q \Phi) A_{1} \cdots A_{n-1}\right\rangle\right\rangle, \\
& \left\langle\left\langle A_{1} \cdots A_{n-1}\left(\eta_{0} \Phi\right)\right\rangle\right\rangle=-\left\langle\left\langle\left(\eta_{0} \Phi\right) A_{1} \cdots A_{n-1}\right\rangle\right\rangle,
\end{aligned}
$$

and an identity $\left[A_{t}^{(0)}, A_{Q}^{(0)}\right]=Q A_{t}^{(0)}-\partial_{t} A_{Q}^{(0)}$. Using $e^{\Phi^{\prime}(t)}\left(\eta_{0} A_{t}^{\prime}\right) e^{-\Phi^{\prime}(t)}=-\partial_{t}\left(e^{\Phi^{\prime}(t)}\left(\eta_{0} e^{-\Phi^{\prime}(t)}\right)\right)$, $Q\left(e^{\Phi^{\prime}(t)}\left(\eta_{0} e^{-\Phi^{\prime}(t)}\right)\right)=e^{\Phi^{\prime}(t)}\left(\eta_{0} A_{Q}^{\prime}\right) e^{-\Phi^{\prime}(t)}$ and partial integrability:

$$
\langle\langle Q(\cdots)\rangle\rangle=0, \quad\left\langle\left\langle\eta_{0}(\cdots)\right\rangle\right\rangle=0
$$

we can simplify the last three lines of (B.7) as:

$$
\begin{aligned}
& \left\langle\left\langle\left(\eta_{0} A_{t}^{\prime}\right) e^{-\Phi^{\prime}(t)} A_{Q}^{(0)} e^{\Phi^{\prime}(t)}-e^{\Phi^{\prime}(t)}\left(\eta_{0} e^{-\Phi^{\prime}(t)}\right) \partial_{t} A_{Q}^{(0)}\right\rangle\right\rangle=-\partial_{t}\left\langle\left\langle e^{\Phi^{\prime}(t)}\left(\eta_{0} e^{-\Phi^{\prime}(t)}\right) A_{Q}^{(0)}\right\rangle\right\rangle,(B) \\
& \left\langle\left\langle\left(\eta_{0} A_{t}^{(0)}\right) e^{\Phi^{\prime}(t)} A_{Q}^{\prime} e^{-\Phi^{\prime}(t)}+\left(\eta_{0} e^{-\Phi^{\prime}(t)}\right) A_{t}^{(0)} e^{\Phi^{\prime}(t)} A_{Q}^{\prime}+e^{-\Phi^{\prime}(t)} A_{t}^{(0)}\left(\eta_{0} e^{\Phi^{\prime}(t)}\right) A_{Q}^{\prime}\right\rangle\right\rangle \\
& \quad=-\left\langle\left\langle A_{t}^{(0)} e^{\Phi^{\prime}(t)}\left(\eta_{0} A_{Q}^{\prime}\right) e^{-\Phi^{\prime}(t)}\right\rangle\right\rangle \\
& \left\langle\left\langle e^{-\Phi^{\prime}(t)}\left(\eta_{0} e^{\Phi^{\prime}(t)}\right)\left(Q A_{t}^{(0)}\right)\right\rangle\right\rangle=\left\langle\left\langle A_{t}^{(0)} e^{\Phi^{\prime}(t)}\left(\eta_{0} A_{Q}^{\prime}\right) e^{-\Phi^{\prime}(t)}\right\rangle\right\rangle .
\end{aligned}
$$

Then we have proved an identity

$$
\left\langle\left\langle\left(\eta_{0} A_{t}\right) A_{Q}\right\rangle\right\rangle=\left\langle\left\langle\left(\eta_{0} A_{t}^{(0)}\right) A_{Q}^{(0)}\right\rangle\right\rangle+\left\langle\left\langle\left(\eta_{0} A_{t}^{\prime}\right) A_{Q}^{\prime}\right\rangle\right\rangle-\partial_{t}\left\langle\left\langle e^{\Phi^{\prime}(t)}\left(\eta_{0} e^{-\Phi^{\prime}(t)}\right) A_{Q}^{(0)}\right\rangle\right\rangle
$$

which implies the action (B.6) is rewritten for $e^{\Phi}=e^{\Phi^{(0)}} e^{\Phi^{\prime}}$ as

$$
S[\Phi]=S\left[\Phi^{(0)}\right]+S\left[\Phi^{\prime}\right]+\frac{1}{g^{2}}\left\langle\left\langle e^{\Phi^{\prime}}\left(\eta_{0} e^{-\Phi^{\prime}}\right) \bar{A}_{Q}^{(0)}\right\rangle\right\rangle,
$$

where we have imposed ordinary boundary conditions $\Phi^{(0)}(1)=\Phi^{(0)}, \Phi^{\prime}(1)=\Phi^{\prime}, \Phi^{(0)}(0)=$ $\Phi^{\prime}(0)=0$ and denoted $\bar{A}_{Q}^{(0)}=\left.A_{Q}^{(0)}\right|_{t=1}$. The last extra term of the above action can be 
rewritten as follows:

$$
\begin{aligned}
& \frac{1}{g^{2}}\left\langle\left\langle e^{\Phi^{\prime}}\left(\eta_{0} e^{-\Phi^{\prime}}\right) \bar{A}_{Q}^{(0)}\right\rangle\right\rangle \\
= & \frac{1}{g^{2}} \int_{0}^{1} d t \partial_{t}\left\langle\left\langle e^{\Phi^{\prime}(t)}\left(\eta_{0} e^{-\Phi^{\prime}(t)}\right) \bar{A}_{Q}^{(0)}\right\rangle\right\rangle=-\frac{1}{g^{2}} \int_{0}^{1} d t\left\langle\left\langle e^{\Phi^{\prime}(t)}\left(\eta_{0} A_{t}^{\prime}\right) e^{-\Phi^{\prime}(t)} \bar{A}_{Q}^{(0)}\right\rangle\right\rangle \\
= & -\frac{1}{g^{2}} \int_{0}^{1} d t\left\langle\left\langle\left(\eta_{0} A_{t}^{\prime}\right)\left(e^{-\Phi^{\prime}(t)} \bar{A}_{Q}^{(0)} e^{\Phi^{\prime}(t)}-\bar{A}_{Q}^{(0)}\right)-A_{t}^{\prime}\left(\eta_{0} \bar{A}_{Q}^{(0)}\right)\right\rangle\right\rangle .
\end{aligned}
$$

In the first equality, we have kept $\bar{A}_{Q}^{(0)}$ intact in $t$-integration and used $e^{\Phi^{\prime}(t)}\left(\eta_{0} A_{t}^{\prime}\right) e^{-\Phi^{\prime}(t)}=$ $-\partial_{t}\left(e^{\Phi^{\prime}(t)}\left(\eta_{0} e^{-\Phi^{\prime}(t)}\right)\right)$ again in the second equality. Using (B.6), (B.16) and (B.17), and imposing equation of motion for $\Phi^{(0)}: \eta_{0} \bar{A}_{Q}^{(0)}=0$, we have obtained the action for $\Phi^{\prime}$ around a classical solution $\Phi^{(0)}$ in the same form as the original one:

$$
S^{\prime}\left[\Phi^{\prime}\right] \equiv S[\Phi]-S\left[\Phi^{(0)}\right]=-\frac{1}{g^{2}} \int_{0}^{1} d t\left\langle\left\langle\left(\eta_{0} A_{t}^{\prime}\right) A_{Q^{\prime}}^{\prime}\right\rangle\right\rangle,
$$

with $A_{Q^{\prime}}^{\prime}=e^{-\Phi^{\prime}(t)}\left(Q^{\prime} e^{\Phi^{\prime}(t)}\right)$, where the new BRS operator $Q^{\prime}$ is given by

$$
Q^{\prime} B=Q B+\bar{A}_{Q}^{(0)} * B-(-1)^{|B|} B * \bar{A}_{Q}^{(0)}, \quad \bar{A}_{Q}^{(0)}=e^{-\Phi^{(0)}}\left(Q e^{\Phi^{(0)}}\right) .
$$

We note that $Q^{\prime}$ is a derivation with respect to the star product, nilpotency $Q^{\prime 2}=0$ holds automatically, and $\left\{Q^{\prime}, \eta_{0}\right\}=0$ is satisfied by equation of motion $\eta_{0} \bar{A}_{Q}^{(0)}=0$ for $\Phi^{(0)}$. The above action can be rewritten in the ordinary WZW form again

$$
S^{\prime}\left[\Phi^{\prime}\right]=-\frac{1}{2 g^{2}}\left\langle\left\langle\bar{A}_{\eta_{0}}^{\prime} \bar{A}_{Q^{\prime}}^{\prime}\right\rangle\right\rangle-\frac{1}{2 g^{2}} \int_{0}^{1} d t\left\langle\left\langle A_{t}^{\prime}\left\{A_{Q^{\prime}}^{\prime}, A_{\eta_{0}}^{\prime}\right\}\right\rangle\right\rangle
$$

where $A_{\eta_{0}}^{\prime}=e^{-\Phi^{\prime}(t)}\left(\eta_{0} e^{\Phi^{\prime}(t)}\right), \bar{A}_{\eta_{0}}^{\prime}=\left.A_{\eta_{0}}^{\prime}\right|_{t=0}, \bar{A}_{Q^{\prime}}^{\prime}=\left.A_{Q^{\prime}}^{\prime}\right|_{t=1}$, using the method in 34].

\section{Supersymmetry in superstring field theories}

First, we will show that the fermionic transformation (2.80), which is generated by (2.79), corresponds to global space-time supersymmetries.

For the parameter (2.79), the transformation law given in (2.77) and (2.78) becomes

$$
\begin{aligned}
\delta_{\epsilon} \Phi & =-\frac{\mathrm{ad}_{\Phi}}{1-e^{-\mathrm{ad}_{\Phi}}}\left\{\Omega(\epsilon), \eta_{0} \Psi\right\}=-\frac{\operatorname{ad}_{\Phi}}{1-e^{-\mathrm{ad}_{\Phi}}}\left(\oint \frac{d z}{2 \pi i} \epsilon_{\alpha} \xi S_{(-1 / 2)}^{\alpha}(z) \eta_{0} \Psi\right), \\
\delta_{\epsilon}\left(\eta_{0} \Psi\right) & =\eta_{0}\left\{\Omega(\epsilon), e^{-\Phi} Q_{\mathrm{B}} e^{\Phi}\right\}=\eta_{0} \oint \frac{d z}{2 \pi i} \epsilon_{\alpha} \xi S_{(-1 / 2)}^{\alpha}(z)\left(e^{-\Phi} Q_{\mathrm{B}} e^{\Phi}\right),
\end{aligned}
$$

where the term $\eta_{0} Q_{\mathrm{B}} \Omega(\epsilon)$ is not included as is explained in footnote 14 in order to express it only in terms of a contour integration (2.81). These transformations are equivalent to (2.80). 
The equations of motion are given by

$$
\begin{aligned}
& f_{1} \equiv \eta_{0}\left(e^{-\Phi} Q_{\mathrm{B}} e^{\Phi}\right)+\left(\eta_{0} \Psi\right)^{2}=0, \\
& f_{2} \equiv e^{-\Phi}\left(Q_{\mathrm{B}}\left(e^{\Phi}\left(\eta_{0} \Psi\right) e^{-\Phi}\right)\right) e^{\Phi}=Q_{\mathrm{B}} \eta_{0} \Psi+\left\{e^{-\Phi} Q_{\mathrm{B}} e^{\Phi}, \eta_{0} \Psi\right\}=0 .
\end{aligned}
$$

Note that the $\mathrm{R}$ sector string field $\Psi$ is involved in the equations of motion through the particular form $\eta_{0} \Psi$. We apply the transformation (2.80) to the string fields $f_{1}$ and $f_{2}$ :

$$
\begin{aligned}
& \delta_{\epsilon} f_{1}=\eta_{0} \mathcal{S}(\epsilon) f_{2} \\
& \delta_{\epsilon} f_{2}=-\left\{Q_{\mathrm{B}}, \mathcal{S}(\epsilon)\right\} f_{1}+\left[f_{1}, \mathcal{S}(\epsilon)\left(e^{-\Phi} Q_{\mathrm{B}} e^{\Phi}\right)\right]+\left\{\mathcal{S}(\epsilon) f_{2}, \eta_{0} \Psi\right\}
\end{aligned}
$$

If $f_{1}=f_{2}=0$, we find $\delta_{\epsilon} f_{1}=\delta_{\epsilon} f_{2}=0$. Hence this symmetry is realized only on-shell.

Let us consider how massless fields are transformed by (2.80). The string fields contain massless fields as follows:

$$
\begin{aligned}
\left|\Phi_{A}\right\rangle & =\int \frac{d^{10} p}{(2 \pi)^{10}}\left(\tilde{A}_{\mu}(p) c \xi e^{-\phi} \psi^{\mu}(0)+\tilde{B}(p) c \partial c \xi \partial \xi e^{-2 \phi}(0)\right)\left|p^{\mu}, q=0\right\rangle \\
\left|\Psi_{\lambda}\right\rangle & =\int \frac{d^{10} p}{(2 \pi)^{10}} \tilde{\lambda}_{\alpha}(p) \xi S_{(-1 / 2)}^{\alpha} c(0)\left|p^{\mu}, q=0\right\rangle
\end{aligned}
$$

where $\tilde{A}_{\mu}(p), \tilde{B}(p)$ and $\tilde{\lambda}_{\alpha}(p)$ denote Fourier transforms of gluon, auxiliary Nakanishi-Lautrup [41] and gluino [32] fields, respectively. We note that $q$ in $\left|p^{\mu}, q\right\rangle$ implies a zero-mode momentum of $\phi$. Applying the transformation (2.80) to these fields, we find

$$
\begin{aligned}
\delta_{\epsilon}\left|\Phi_{A}\right\rangle= & \oint \frac{d z}{2 \pi i} \epsilon_{\alpha} \xi S_{(-1 / 2)}^{\alpha}(z) \eta_{0}\left|\Psi_{\lambda}\right\rangle+\cdots \\
= & \int \frac{d^{10} p}{(2 \pi)^{10}}\left(-i \epsilon_{\alpha}\left(\Gamma_{\mu} C\right)^{\alpha \beta} \tilde{\lambda}_{\beta}(p)\right) c \xi e^{-\phi} \psi^{\mu}(0)\left|p^{\mu}, 0\right\rangle+\cdots \\
\delta_{\epsilon}\left(\eta_{0}\left|\Psi_{\lambda}\right\rangle\right)= & \eta_{0} \oint \frac{d z}{2 \pi i} \epsilon_{\alpha} \xi S_{(-1 / 2)}^{\alpha}(z) Q_{\mathrm{B}}\left|\Phi_{A}\right\rangle+\cdots \\
= & \int \frac{d^{10} p}{(2 \pi)^{10}} \epsilon_{\alpha}\left(\frac{1}{4} \sqrt{2 \alpha^{\prime}}\left(p_{\mu} \tilde{A}_{\nu}(p)-p_{\nu} \tilde{A}_{\mu}(p)\right)\left(\Gamma^{\mu \nu}\right)_{\beta}^{\alpha}\right. \\
& \left.+\left(\sqrt{\frac{\alpha^{\prime}}{2}} p^{\mu} \tilde{A}_{\mu}(p)+\tilde{B}(p)\right) \delta_{\beta}^{\alpha}\right) S_{(-1 / 2)}^{\beta} c(0)\left|p^{\mu}, 0\right\rangle+\cdots
\end{aligned}
$$

where $(+\cdots)$ denotes quadratic or higher order terms with respect to component fields. Here we have calculated the above results by using the OPEs ${ }^{23}$

$$
S_{(-1 / 2)}^{\alpha}(y) S_{(-1 / 2)}^{\beta}(z) \sim \frac{1}{y-z} i\left(\Gamma_{\mu} C\right)^{\alpha \beta} \psi^{\mu} e^{-\phi}(z),
$$

\footnotetext{
${ }^{23} \mathrm{We}$ have used the convention in [58] for spin fields and taken $T^{\mathrm{m}}(z)=-\frac{1}{4 \alpha^{\prime}} \partial X_{\mu} \partial X^{\mu}(z)-$ $\frac{1}{2} \psi^{\mu} \partial \psi_{\mu}(z), G^{\mathrm{m}}(z)=\frac{i}{\sqrt{2 \alpha^{\prime}}} \psi^{\mu} \partial X_{\mu}(z)$ with $X^{\mu}(y) X^{\nu}(z) \sim-2 \alpha^{\prime} \eta^{\mu \nu} \log (y-z), \psi^{\mu}(y) \psi^{\nu}(z) \sim \eta^{\mu \nu}(y-z)^{-1}$ in the matter sector and $j_{\mathrm{B}}(z)=c\left(T^{\mathrm{m}}-\frac{1}{2}(\partial \phi)^{2}-\partial^{2} \phi+\partial \xi \eta\right)(z)+b c \partial c(z)+\eta e^{\phi} G^{\mathrm{m}}(z)-\eta \partial \eta e^{2 \phi} b(z)+\partial^{2} c(z)+$ $\partial(c \xi \eta)(z)$ for the BRS current.
} 


$$
\begin{aligned}
& j_{\mathrm{B}}(y) c \xi e^{-\phi} \psi^{\mu} e^{i p_{\nu} X^{\nu}}(z) \sim \frac{1}{(y-z)^{2}} \sqrt{2 \alpha^{\prime}} p^{\mu} c e^{i p_{\nu} X^{\nu}}(z)+\frac{1}{y-z}\left(\frac{i}{\sqrt{2 \alpha^{\prime}}} c \partial X^{\mu}+\eta c e^{\phi} \psi^{\mu}\right. \\
& \left.+\sqrt{2 \alpha^{\prime}} c\left(p_{\nu} \psi^{\nu} \psi^{\mu}+p^{\mu}(\partial \phi-\xi \eta)\right)-\alpha^{\prime} p^{2} c \partial c \xi e^{-\phi} \psi^{\mu}\right) e^{i p_{\nu} X^{\nu}}(z) \\
& j_{\mathrm{B}}(y) c \partial c \xi \partial \xi e^{-2 \phi} e^{i p_{\nu} X^{\nu}}(z) \sim \frac{1}{(y-z)^{2}} c e^{i p_{\nu} X^{\nu}}(z) \\
& +\frac{1}{y-z}\left(-\partial c+2 c(\partial \phi-\xi \eta)+\sqrt{2 \alpha^{\prime}} c \partial c \xi p_{\mu} \psi^{\mu} e^{-\phi}\right) e^{i p_{\nu} X^{\nu}}(z) \\
& \xi S_{(-1 / 2)}^{\alpha}(y) c(\partial \phi-\xi \eta)(z) \sim \frac{1}{y-z}\left(\frac{1}{2} \xi S_{(-1 / 2)}^{\alpha} c\right)(z) \\
& \xi S_{(-1 / 2)}^{\alpha}(y) c \psi^{\nu} \psi^{\mu}(z) \sim \frac{1}{y-z} \frac{1}{2} \xi\left(\Gamma^{\nu \mu} S_{(-1 / 2)}\right)^{\alpha} c(z) .
\end{aligned}
$$

From (C.9) and (C.11), we can read off the transformation law for massless fields:

$$
\begin{aligned}
\delta_{\epsilon} \tilde{A}_{\mu}(p) & =-i \epsilon \Gamma_{\mu} C \tilde{\lambda}(p)+\cdots \\
\delta_{\epsilon} \tilde{B}(p) & =0+\cdots \\
\delta_{\epsilon} \tilde{\lambda}_{\alpha}(p) & =-\frac{\sqrt{2 \alpha^{\prime}}}{4}\left(p_{\mu} \tilde{A}_{\nu}(p)-p_{\nu} \tilde{A}_{\mu}(p)\right)\left(\epsilon \Gamma^{\mu \nu}\right)_{\alpha}-\left(\sqrt{\frac{\alpha^{\prime}}{2}} p^{\mu} \tilde{A}_{\mu}(p)+\tilde{B}(p)\right) \epsilon_{\alpha}+\cdots
\end{aligned}
$$

For massless fields, the linearized equations of motion are calculated as

$$
\begin{aligned}
Q_{\mathrm{B}} \eta_{0}\left|\Phi_{A}\right\rangle= & \int \frac{d^{10} p}{(2 \pi)^{10}}\left(-\left(\sqrt{2 \alpha^{\prime}} p^{\mu} \tilde{A}_{\mu}(p)+2 \tilde{B}(p)\right) c \eta(0)\right. \\
& \left.+\left(\alpha^{\prime} p^{2} \tilde{A}(p)+\sqrt{2 \alpha^{\prime}} p_{\mu} \tilde{B}(p)\right) c \partial c e^{-\phi} \psi^{\mu}(0)\right)\left|p^{\nu}, 0\right\rangle=0 \\
Q_{\mathrm{B}} \eta_{0}\left|\Psi_{\lambda}\right\rangle= & \int \frac{d^{10} p}{(2 \pi)^{10}}\left(\alpha^{\prime} p^{2} \tilde{\lambda}_{\alpha}(p) S_{(-1 / 2)}^{\alpha} c \partial c(0)\right. \\
& \left.+\sqrt{\alpha^{\prime}} i p_{\mu} \tilde{\lambda}_{\alpha}(p)\left(\Gamma^{\mu}\right)^{\alpha} S_{(1 / 2)} S_{(\dot{\beta}} \eta c(0)\right)\left|p^{\nu}, 0\right\rangle=0
\end{aligned}
$$

where $S_{(1 / 2)}^{\dot{\beta}}$ is the $\operatorname{GSO}(+)$ spin operator with $\phi$-charge $1 / 2$, dimension 0 and negative chirality. Consequently, we obtain the linearized equations of motion:

$$
\begin{aligned}
& \tilde{B}(p)=-\sqrt{\frac{\alpha^{\prime}}{2}} p^{\mu} \tilde{A}_{\mu}(p), \quad\left(p^{2} \delta_{\mu}^{\nu}-p_{\mu} p^{\nu}\right) \tilde{A}_{\nu}(p)=0, \\
& \tilde{\lambda}_{\alpha}(p) p^{2}=0, \quad \tilde{\lambda}(p) \Gamma^{\mu} p_{\mu}=0 .
\end{aligned}
$$

Under these on-shell conditions, the transformation laws (C.17), (C.18) and (C.19) become

$$
\delta_{\epsilon} A_{\mu}=-i \epsilon \Gamma_{\mu} C \lambda, \quad \delta_{\epsilon} \lambda=\frac{i}{2} \sqrt{\frac{\alpha^{\prime}}{2}} F_{\mu \nu}\left(\epsilon \Gamma^{\mu \nu}\right), \quad\left(F_{\mu \nu}=\partial_{\mu} A_{\nu}-\partial_{\nu} A_{\mu}\right) .
$$

These are nothing but supersymmetry transformation of 10D supersymmetric Maxwell theory. Hence, the transformation law (2.80) contains space-time supersymmetries.

Finally, we would like to comment on supersymmetry in the cubic open superstring field theory [30] and the modified cubic theory [35, 36]. 
Cubic version In the Witten's open superstring field theory, there are fermionic gauge symmetry and global supersymmetry at least formally [30. Fermionic gauge symmetry, which is generated by Grassmann even gauge parameter $\chi$ in the Ramond sector with picture number $-1 / 2$ and ghost number 0 , is given by

$$
\begin{aligned}
& \delta_{\chi} A=\Psi * \chi-\chi * \Psi, \\
& \delta_{\chi} \Psi=Q_{\mathrm{B}} \chi+X(i)(A * \chi-\chi * A),
\end{aligned}
$$

where $X(i)$ is the picture changing operator at the midpoint, and $A(\Psi)$ denotes a Grassmann odd string field in the NS (R) sector with picture number $-1(-1 / 2)$ and ghost number 1 (1). Formally, by taking

$$
\chi=-\int_{C_{\text {left }}} \frac{d z}{2 \pi i} \epsilon_{\alpha} S_{(-1 / 2)}^{\alpha}(z) I,
$$

and omitting $Q_{\mathrm{B}} \chi=\frac{1}{2 \pi i} \epsilon_{\alpha}\left(c S_{(-1 / 2)}^{\alpha}(i)-c S_{(-1 / 2)}^{\alpha}(-i)\right) I$ in $\delta_{\chi} \Psi$, which is itself a symmetry of the action, the above gauge transformation becomes global space-time supersymmetry transformation:

$$
\begin{aligned}
\delta_{\epsilon} A & =\oint \frac{d z}{2 \pi i} \epsilon_{\alpha} S_{(-1 / 2)}^{\alpha}(z) \Psi \\
\delta_{\epsilon} \Psi & =X(i) \oint \frac{d z}{2 \pi i} \epsilon_{\alpha} S_{(-1 / 2)}^{\alpha}(z) A .
\end{aligned}
$$

Modified cubic version In modified version of cubic open superstring field theory, there are also fermionic gauge symmetry and global supersymmetry. Fermionic gauge symmetry, which is generated by Grassmann even gauge parameter $\chi$ in the Ramond sector with picture number $-1 / 2$ and ghost number 0 , is given by

$$
\begin{aligned}
& \delta_{\chi} A=X(i)(\Psi * \chi-\chi * \Psi), \\
& \delta_{\chi} \Psi=Q_{\mathrm{B}} \chi+A * \chi-\chi * A,
\end{aligned}
$$

where $A(\Psi)$ denotes a Grassmann odd string field in the NS (R) sector with picture number $0(-1 / 2)$ and ghost number 1 (1). Formally, by taking

$$
\begin{aligned}
\chi= & -Y(i) \int_{C_{\text {left }}} \frac{d z}{2 \pi i} Q_{\mathrm{B}} \epsilon_{\alpha} \xi S_{(-1 / 2)}^{\alpha}(z) I=Y(i) \int_{C_{\text {left }}} \frac{d z}{2 \pi i} \epsilon_{\alpha} W^{\alpha}(z) I, \\
& W^{\alpha}(z) \equiv\left[Q_{\mathrm{B}}, \xi S_{(-1 / 2)}^{\alpha}(z)\right],
\end{aligned}
$$


with the inverse picture changing operator $Y(z)$, which is global: $Q_{\mathrm{B}} \chi=0$, the above gauge transformation yields the global space-time supersymmetry transformation [59]: ${ }^{24}$

$$
\begin{aligned}
\delta_{\epsilon} A & =\oint \frac{d z}{2 \pi i} \epsilon_{\alpha} W^{\alpha}(z) \Psi \\
\delta_{\epsilon} \Psi & =Y(i) \oint \frac{d z}{2 \pi i} \epsilon_{\alpha} W^{\alpha}(z) A .
\end{aligned}
$$

\section{Classical solutions and marginal deformations in Wit- ten's bosonic open string field theory}

In this section, we consider classical solutions of the Witten's bosonic string field theory corresponding to marginal deformations, which are generalization of the previous ones investigated in [21] $]^{25}$ and counterparts of the arguments in \$3. We discuss a class of solutions using a current $J^{a}$ associated with a Lie algebra $\mathcal{G}$. We suppose the OPE among currents with adjoint indices of the form

$$
\begin{aligned}
J^{a}(y) J^{b}(z) & \sim-g^{a b} \frac{1}{(y-z)^{2}}+\frac{1}{y-z} f_{c}^{a b} J^{c}(z), \\
g^{a b} & =\frac{1}{2}\left(f_{d}^{a c} f_{c}^{b d}-\Omega^{a b}\right),
\end{aligned}
$$

where $f_{c}^{a b}$ is the structure constant of $\mathcal{G}$ and $\Omega^{a b}$ is a particular invertible invariant matrix such as (3.4). $J^{a}$ is a primary field with dimension 1 for the energy momentum tensor given by the Sugawara form:

$$
T(z)=\Omega_{a b}: J^{a} J^{b}:(z), \quad\left(\Omega^{a b} \Omega_{b c}=\delta_{c}^{a}\right) .
$$

In fact, we can show the OPEs

$$
\begin{aligned}
T(y) J^{a}(z) & \sim \frac{1}{(y-z)^{2}} J^{a}(z)+\frac{1}{y-z} \partial J^{a}(z) \\
T(y) T(z) & \sim \frac{c}{2} \frac{1}{(y-z)^{4}}+\frac{1}{(y-z)^{2}} 2 T(z)+\frac{1}{y-z} \partial T(z),
\end{aligned}
$$

where the central charge $c$ of the Virasoro algebra is given by $c=\operatorname{dim\mathcal {G}}-f_{d}^{a c} f_{c}^{b d} \Omega_{a b}$ [45]. In the following, we assume that the background is described by the above CFT with $c=26$, and construct a classical solution of bosonic open string field theory of cubic form:

$$
S[\Psi]=-\frac{1}{g^{2}}\left(\frac{1}{2}\left\langle\Psi Q_{\mathrm{B}} \Psi\right\rangle+\frac{1}{3}\langle\Psi \Psi \Psi\rangle\right)
$$

\footnotetext{
${ }^{24}$ Similar formula can be found in [36, 60. See also 61].

${ }^{25}$ General arguments in bosonic string field theory are given in 62 .
} 
on this background. Namely, the BRS operator in the kinetic term is

$$
Q_{\mathrm{B}}=\oint \frac{d z}{2 \pi i}(c T(z)+b c \partial c(z))
$$

where $T(z)$ is given by eq. (D.3) and interaction term is defined by the Witten $*$ product using conformal mappings and CFT correlators $[38,63$. With the above setup, we can show commutation relations

$$
\begin{aligned}
& \left\{Q_{\mathrm{B}}, c J^{a}(z)\right\}=0, \quad\left\{Q_{\mathrm{B}}, c(z)\right\}=c \partial c(z), \\
& \left\{V_{L}^{a}(f), V_{L}^{b}(g)\right\}=\frac{1}{2} g^{a b}\left\{Q_{\mathrm{B}}, C_{L}(f g)\right\}, \quad\left\{V_{L}^{a}(f), C_{L}(g)\right\}=\left\{C_{L}(f), C_{L}(g)\right\}=0,
\end{aligned}
$$

where $V_{L}^{a}(f)=\int_{C_{\text {left }}} \frac{d z}{2 \pi i} \frac{1}{\sqrt{2}} f(z) c J^{a}(z)$ and $C_{L}(f)=\int_{C_{\text {left }}} \frac{d z}{2 \pi i} f(z) c(z)$ using similar method in [22]. Then, noting $c J^{a}(z)$ is a primary field with dimension 0 , we have

$$
V_{L}^{a}\left(F_{a}\right) I * V_{L}^{b}\left(F_{b}\right) I=V_{L}^{a}\left(F_{a}\right) V_{L}^{b}\left(F_{b}\right) I=\frac{1}{4} g^{a b}\left\{Q_{\mathrm{B}}, C_{L}\left(F_{a} F_{b}\right)\right\} I,
$$

with $F_{a}(-1 / z)=z^{2} F_{a}(z)$. Using this relation, we can show that

$$
\Psi_{0}=-V_{L}^{a}\left(F_{a}\right) I-\frac{1}{4} g^{a b} C_{L}\left(F_{a} F_{b}\right) I, \quad F_{a}(-1 / z)=z^{2} F_{a}(z),
$$

satisfies the equation of motion: $Q_{\mathrm{B}} \Psi_{0}+\Psi_{0} * \Psi_{0}=0$. If we re-expand the action around this solution such as $\Psi=\Psi_{0}+\Psi^{\prime}$, we have $S[\Psi]=S\left[\Psi_{0}\right]+S^{\prime}\left[\Psi^{\prime}\right]$ where the new action $S^{\prime}\left[\Psi^{\prime}\right]$ is the same form as original one (D.6) except that the new BRS operator is given by $Q_{\mathrm{B}}^{\prime} A=Q_{\mathrm{B}} A+\Psi_{0} * A-(-1)^{|A|} A * \Psi_{0}$, or more explicitly:

$$
Q_{\mathrm{B}}^{\prime}=Q_{\mathrm{B}}-V^{a}\left(F_{a}\right)-\frac{1}{4} g^{a b} C\left(F_{a} F_{b}\right),
$$

$\left(V^{a}(f)=\oint \frac{d z}{2 \pi i} \frac{1}{\sqrt{2}} f(z) c J^{a}(z), C(f)=\oint \frac{d z}{2 \pi i} f(z) c(z)\right)$. Comparing this $Q_{\mathrm{B}}^{\prime}$ with original one $Q_{\mathrm{B}}$ (D.7), we find the Virasoro operator $T(z)$ in the matter sector is replaced by $T(z)-$ $\frac{1}{\sqrt{2}} F_{a}(z) J^{a}(z)-\frac{1}{4} g^{a b} F_{a}(z) F_{b}(z)$. In fact, if we define $T^{\prime}(z)=\sum_{n} L_{n}^{\prime} z^{-n-2}$ as

$$
L_{n}^{\prime}=L_{n}-\frac{1}{\sqrt{2}} \sum_{k} F_{a, k} J_{n-k}^{a}-\frac{1}{4} g^{a b} \sum_{k} F_{a, n-k} F_{b, k},
$$

with $F_{a, n}=\oint \frac{d \sigma}{2 \pi} e^{i(n+1) \sigma} F_{a}\left(e^{i \sigma}\right)$, we obtain the Virasoro algebra with the same central charge $c=26$ as the original one:

$$
\left[L_{m}^{\prime}, L_{n}^{\prime}\right]=(m-n) L_{m+n}^{\prime}+\frac{c}{12}\left(m^{3}-m\right) \delta_{m+n, 0} .
$$


Furthermore, by taking $J^{\prime a}(z)=\sum_{n} J_{n}^{\prime a} z^{-n-1} \operatorname{as}^{26}$

$$
\begin{aligned}
& J_{n}^{\prime a}=\sum_{k} M_{b, k}^{a}\left(J_{n-k}^{b}+\frac{1}{\sqrt{2}} g^{b c} F_{c, n-k}\right), \\
& \sum_{n} M_{b, n}^{a} e^{-i n \sigma}=\left[\mathbf{P} \exp \left(i \int_{0}^{1} d t \sigma A(t \sigma)\right)\right]_{b}^{a}, \quad A_{b}^{a}(\sigma)=\frac{1}{\sqrt{2}} f_{b}^{a c} e^{i \sigma} F_{c}\left(e^{i \sigma}\right),
\end{aligned}
$$

the same commutation relations as the original one are recovered:

$$
\left[J_{m}^{\prime a}, J_{n}^{\prime b}\right]=-g^{a b} m \delta_{m+n, 0}+f_{c}^{a b} J_{m+n}^{\prime c}, \quad\left[L_{m}^{\prime}, J_{n}^{\prime a}\right]=-n J_{m+n}^{\prime a}
$$

In the above, we have constructed a classical solution $\Psi_{0}$ (D.11) and re-expanded around it. The obtained action $S^{\prime}\left[\Psi^{\prime}\right]$ is also reproduced by replacing $T(z)$ with $T^{\prime}(z)(\mathrm{D} .13)$ in the original action $S[\Psi](\underline{\mathrm{D} .6})$. This replacement is induced by the map $J^{a} \rightarrow J^{\prime a}(\underline{\mathrm{D} .15})$ in terms of the current which preserves the algebra among $\left(J^{a}(z), T(z)\right)$. At least formally, we can show that the vacuum energy vanishes at this solution [22, 47]:

$$
S\left[\Psi_{0}\right]=\int_{0}^{1} d t \frac{d}{d t} S\left[\Psi_{0}(t)\right]=-\frac{1}{g^{2}} \int_{0}^{1} d t\left\langle\frac{d}{d t} \Psi_{0}(t)\left(Q_{\mathrm{B}} \Psi_{0}(t)+\Psi_{0}(t) * \Psi_{0}(t)\right)\right\rangle=0
$$

where $\Psi_{0}(t)$ is given by replacing $F_{a}(z)$ with $t F_{a}(z)$ in $\Psi_{0}(\mathrm{D} .11)$. These facts suggest that $\Psi_{0}(\underline{\mathrm{D} .11})$ may be a pure gauge solution. In fact, noting $\left[Q_{\mathrm{B}}, J^{a}(z)\right]=\partial\left(c J^{a}\right)(z)$, we make an ansatz for the gauge parameter as

$$
\Lambda_{L} I=\int_{C_{\mathrm{left}}} \frac{d z}{2 \pi i} \frac{1}{\sqrt{2}} g_{a}(z) J^{a}(z) I ; \quad g_{a}(-1 / z)=g_{a}(z), \quad g_{a}( \pm i)=0 .
$$

Using the OPE (D.1), we can compute its pure gauge form as

$$
\begin{aligned}
& e^{-\Lambda_{L} I} Q_{\mathrm{B}} e^{\Lambda_{L} I} \\
= & -V_{L}^{b}\left(\partial g_{a}\left(\left(e^{\mathcal{M}}-1\right) \mathcal{M}^{-1}\right)_{b}^{a}\right) I-\frac{1}{4} C\left(\partial g_{a} \partial g_{b}\left(\left(e^{\mathcal{M}}-1\right) \mathcal{M}^{-1}\right)^{a}{ }_{c} g^{c d}\left(\left(e^{\mathcal{M}}-1\right) \mathcal{M}^{-1}\right)^{b}{ }_{d}\right) I,
\end{aligned}
$$

where we denoted $\mathcal{M}_{b}^{a}(z)=\frac{1}{\sqrt{2}} f_{b}^{a c} g_{c}(z)$. Comparing with the solution (D.11), we can get a gauge parameter by solving

$$
F_{b}(z)=\partial g_{a}(z)\left(\left(e^{\mathcal{M}}-1\right) \mathcal{M}^{-1}\right)_{b}^{a}(z)
$$

with respect to $g_{a}(z)$ for a given $F_{a}(z)$. For a solution $g_{a}(z)$, the new BRS operator $Q_{\mathrm{B}}^{\prime}(\mathrm{D} .12)$ can be rewritten as a similarity transformation from the original one:

$$
Q_{\mathrm{B}}^{\prime}=e^{-\Lambda} Q_{\mathrm{B}} e^{\Lambda}, \quad \Lambda=\oint \frac{d z}{2 \pi i} \frac{1}{\sqrt{2}} g_{a}(z) J^{a}(z) .
$$

\footnotetext{
${ }^{26}$ In the definition of $M_{b, n}^{a}$, we use path-ordered form in the same way as eq. (3.36). Therefore, we have similar formulae to eqs. (3.38), (3.39) and (3.40) by replacing $\Omega^{a b}$ with $-2 g^{a b}$ in the bosonic case. Note that $f^{a b} g^{c d}+f_{c}^{a d} g^{c b}=0$.
} 
In this case, we recover the original SFT action by performing a field redefinition such as $\Psi^{\prime \prime}=e^{\Lambda} \Psi^{\prime}$, which implies the effect of a marginal deformation by a current $J^{a}$. Its deformation parameter $g_{a}(z)$ is related to the classical solution of string field theory as (D.21). However, we cannot always obtain a solution $g_{a}(z)$ to the above differential equation (D.21) because of the boundary condition $g_{a}( \pm i)=0$, which is imposed by a partial integration in computing eq. (D.20). This situation is just the same as supersymmetric case in 93 Namely, consistency condition for the pure gauge form (D.20) is given by (3.60) because the differential equation (D.21) is the same as (3.54). Therefore, there is a possibility that a solution $\Psi_{0}$ becomes nontrivial if $F_{a}(z)$ does not satisfy eq. (3.60).

As an example of $\mathcal{G}=u(1)^{26}$, we take the current $J^{\mu}=\frac{i}{\sqrt{2 \alpha^{\prime}}} \partial X^{\mu}$ on the flat background. In this case, we can identify as

$$
T(z)=-\frac{1}{4 \alpha^{\prime}} \partial X^{\mu} \partial X_{\mu}(z), \quad f_{c}^{a b}=0, \quad \Omega^{\mu \nu}=2 \eta^{\mu \nu}, \quad c=\operatorname{dim}\left(u(1)^{26}\right)=26,
$$

using the above notation. If some directions are compactified to the torus, a solution $\Psi_{0}$ (D.11) becomes nontrivial according to a nontrivial Wilson lines: $\int_{C_{\text {left }}} d z F_{\mu}(z) \neq 0$ [21]. When one direction $\left(X^{25}\right)$ is $S^{1}$-compactified at the critical radius $R=\sqrt{\alpha^{\prime}}$, we can regard the algebra as $\mathcal{G}=u(1)^{25} \times s u(2)$ and identify as

$$
\begin{aligned}
& J^{1}(z)=\sqrt{2} \cos \left(\frac{X^{25}}{\sqrt{\alpha^{\prime}}}\right)(z), \quad J^{2}(z)=\sqrt{2} \sin \left(\frac{X^{25}}{\sqrt{\alpha^{\prime}}}\right)(z), \quad J^{3}(z)=\frac{i}{\sqrt{2 \alpha^{\prime}}} \partial X^{25}(z), \\
& T(z)=-\frac{1}{4 \alpha^{\prime}} \partial X^{25} \partial X^{25}(z)=\frac{1}{6}:\left(J^{1} J^{1}+J^{2} J^{2}+J^{3} J^{3}\right):(z), \\
& f_{c}^{a b}=\sqrt{2} i \epsilon_{a b c}, \quad \Omega^{a b}=6 \delta^{a b}, \quad c=\operatorname{dim}(\operatorname{su}(2))-3 \cdot 4 / 6=1,
\end{aligned}
$$

in the $s u(2)$ sector. The corresponding solution $\Psi_{0}$ was investigated in refs. [21, 23, 64].

\section{References}

[1] A. Sen, "Descent relations among bosonic D-branes," Int. J. Mod. Phys. A 14, 4061 (1999) arXiv:hep-th/9902105.

[2] A. Sen, "Universality of the tachyon potential," JHEP 9912, 027 (1999) arXiv:hep-th/9911116.

[3] A. Sen and B. Zwiebach, "Tachyon condensation in string field theory," JHEP 0003, 002 (2000) arXiv:hep-th/9912249. 
[4] A. Sen, "Tachyon dynamics in open string theory," arXiv:hep-th/0410103, and references therein.

[5] N. Berkovits, "SuperPoincare invariant superstring field theory," Nucl. Phys. B 450, 90 (1995) [Erratum-ibid. B 459, 439 (1996)] arXiv:hep-th/9503099.

[6] N. Berkovits, "The tachyon potential in open Neveu-Schwarz string field theory," JHEP 0004, 022 (2000) arXiv:hep-th/0001084.

[7] C. Wendt, "Scattering Amplitudes And Contact Interactions In Witten's Superstring Field Theory," Nucl. Phys. B 314, 209 (1989).

[8] N. Berkovits, A. Sen and B. Zwiebach, "Tachyon condensation in superstring field theory," Nucl. Phys. B 587, 147 (2000) arXiv:hep-th/0002211.

[9] P. J. De Smet and J. Raeymaekers, "Level four approximation to the tachyon potential in superstring field theory," JHEP 0005, 051 (2000) arXiv:hep-th/0003220.

[10] A. Iqbal and A. Naqvi, "Tachyon condensation on a non-BPS D-brane," arXiv:hep-th/0004015.

[11] K. Ohmori, "Tachyonic kink and lump-like solutions in superstring field theory," JHEP 0105, 035 (2001) arXiv:hep-th/0104230.

[12] J. Kluson, "Some solutions of Berkovits' superstring field theory," arXiv:hep-th/0201054.

[13] J. Kluson, "Proposal for background independent Berkovits' superstring field theory," JHEP 0107, 039 (2001) arXiv:hep-th/0106107.

[14] M. Sakaguchi, "Pregeometrical formulation of Berkovits' open RNS superstring field theories," arXiv:hep-th/0112135.

[15] I. Y. Arefeva, A. A. Giryavets and P. B. Medvedev, "NS matter sliver," Phys. Lett. B 532, 291 (2002) arXiv:hep-th/0112214.

[16] M. Marino and R. Schiappa, "Towards vacuum superstring field theory: The supersliver," J. Math. Phys. 44, 156 (2003) arXiv:hep-th/0112231.

[17] I. Y. Arefeva, D. M. Belov and A. A. Giryavets, "Construction of the vacuum string field theory on a non-BPS brane," JHEP 0209, 050 (2002) arXiv:hep-th/0201197. 
[18] I. Y. Arefeva, A. A. Giryavets and A. S. Koshelev, "NS ghost slivers," Phys. Lett. B 536, 138 (2002) arXiv:hep-th/0203227.

[19] K. Ohmori, "Comments on solutions of vacuum superstring field theory," JHEP 0204, 059 (2002) arXiv:hep-th/0204138.

[20] O. Lechtenfeld, A. D. Popov and S. Uhlmann, "Exact solutions of Berkovits' string field theory," Nucl. Phys. B 637, 119 (2002) arXiv:hep-th/0204155.

[21] T. Takahashi and S. Tanimoto, "Wilson lines and classical solutions in cubic open string field theory," Prog. Theor. Phys. 106, 863 (2001) arXiv:hep-th/0107046.

[22] T. Takahashi and S. Tanimoto, "Marginal and scalar solutions in cubic open string field theory," JHEP 0203, 033 (2002) arXiv:hep-th/0202133.

[23] F. Katsumata, T. Takahashi and S. Zeze, "Marginal Deformations and Closed String Couplings in Open String Field Theory," JHEP 0411, 050 (2004) arXiv:hep-th/0409249.

[24] A. Sen and B. Zwiebach, "Large marginal deformations in string field theory," JHEP 0010, 009 (2000) arXiv:hep-th/0007153.

[25] W. Taylor, "D-brane effective field theory from string field theory," Nucl. Phys. B 585, 171 (2000) arXiv:hep-th/0001201.

[26] A. Iqbal and A. Naqvi, "On marginal deformations in superstring field theory," JHEP 0101, 040 (2001) arXiv:hep-th/0008127.

[27] E. Coletti, I. Sigalov and W. Taylor, "Abelian and nonabelian vector field effective actions from string field theory," JHEP 0309, 050 (2003) arXiv:hep-th/0306041.

[28] H. Yang and B. Zwiebach, "Testing closed string field theory with marginal fields," JHEP 0506, 038 (2005) arXiv:hep-th/0501142.

[29] H. Yang and B. Zwiebach, "Dilaton deformations in closed string field theory," JHEP 0505, 032 (2005) arXiv:hep-th/0502161.

[30] E. Witten, "Interacting Field Theory Of Open Superstrings," Nucl. Phys. B 276, 291 (1986). 
[31] Y. Igarashi, K. Itoh, F. Katsumata, T. Takahashi and S. Zeze, "Exploring Vacuum Manifold of Open String Field Theory," arXiv:hep-th/0506083.

[32] N. Berkovits, "The Ramond sector of open superstring field theory," JHEP 0111, 047 (2001) arXiv:hep-th/0109100.

[33] Y. Michishita, "A covariant action with a constraint and Feynman rules for fermions in open superstring field theory," JHEP 0501, 012 (2005) arXiv:hep-th/0412215.

[34] N. Berkovits, Y. Okawa and B. Zwiebach, "WZW-like action for heterotic string field theory," JHEP 0411, 038 (2004) arXiv:hep-th/0409018.

[35] C. R. Preitschopf, C. B. Thorn and S. A. Yost, "Superstring Field Theory," Nucl. Phys. B 337, 363 (1990).

[36] I. Y. Arefeva, P. B. Medvedev and A. P. Zubarev, "New Representation For String Field Solves The Consistence Problem For Open Superstring Field," Nucl. Phys. B 341, 464 (1990).

[37] I. Kishimoto and T. Takahashi, "Open string field theory around universal solutions," Prog. Theor. Phys. 108, 591 (2002) arXiv:hep-th/0205275.

[38] A. LeClair, M. E. Peskin and C. R. Preitschopf, "String Field Theory On The Conformal Plane. 1. Kinematical Principles", Nucl. Phys. B 317, 411 (1989).

[39] A. LeClair, M. E. Peskin and C. R. Preitschopf, "String Field Theory On The Conformal Plane. 2. Generalized Gluing", Nucl. Phys. B 317, 464 (1989).

[40] T. Takahashi, "Tachyon condensation and universal solutions in string field theory," Nucl. Phys. B 670, 161 (2003) arXiv:hep-th/0302182.

[41] N. Berkovits and M. Schnabl, "Yang-Mills action from open superstring field theory," JHEP 0309, 022 (2003) arXiv:hep-th/0307019.

[42] D. J. Gross and A. Jevicki, "Operator Formulation Of Interacting String Field Theory," Nucl. Phys. B 283, 1 (1987).

[43] K. Itoh, K. Ogawa and K. Suehiro, "BRS Invariance Of Witten's Type Vertex," Nucl. Phys. B 289, 127 (1987). 
[44] N. Ohta, "Covariant Interacting String Field Theory In The Fock Space Representation," Phys. Rev. D 34, 3785 (1986) [Erratum-ibid. D 35, 2627 (1987)].

[45] N. Mohammedi, "On bosonic and supersymmetric current algebras for nonsemisimple groups," Phys. Lett. B 325, 371 (1994) arXiv:hep-th/9312182.

[46] A. Recknagel and V. Schomerus, "Boundary deformation theory and moduli spaces of D-branes," Nucl. Phys. B 545, 233 (1999) arXiv:hep-th/9811237.

[47] T. Kugo and B. Zwiebach, "Target space duality as a symmetry of string field theory," Prog. Theor. Phys. 87, 801 (1992) arXiv:hep-th/9201040.

[48] B. Zwiebach, "Interpolating string field theories," Mod. Phys. Lett. A 7, 1079 (1992) arXiv:hep-th/9202015.

[49] Y. Michishita, "On-shell gauge invariants and field strengths in open superstring field theory," Nucl. Phys. B 698, 111 (2004) arXiv:hep-th/0406242.

[50] H. Hata and S. Moriyama, "Boundary and midpoint behaviors of lump solutions in vacuum string field theory," Phys. Rev. D 72, 046004 (2005) arXiv:hep-th/0504184.

[51] D. Friedan, E. J. Martinec and S. H. Shenker, "Conformal Invariance, Supersymmetry And String Theory," Nucl. Phys. B 271, 93 (1986).

[52] A. S. Schwarz and A. Sen, "Gluing Theorem, Star Product And Integration In Open String Field Theory In Arbitrary Background Fields," Int. J. Mod. Phys. A 6, 5387 (1991).

[53] I. Kishimoto and K. Ohmori, "CFT description of identity string field: Toward derivation of the VSFT action,” JHEP 0205, 036 (2002) arXiv:hep-th/0112169.

[54] L. Rastelli and B. Zwiebach, "Tachyon potentials, star products and universality," JHEP 0109, 038 (2001) arXiv:hep-th/0006240.

[55] D. J. Gross and A. Jevicki, "Operator Formulation Of Interacting String Field Theory. 2," Nucl. Phys. B 287, 225 (1987).

[56] D. J. Gross and A. Jevicki, "Operator Formulation Of Interacting String Field Theory. 3. NSR Superstring," Nucl. Phys. B 293, 29 (1987). 
[57] M. Schnabl, "Wedge states in string field theory," JHEP 0301, 004 (2003) arXiv:hep-th/0201095.

[58] V. A. Kostelecky, O. Lechtenfeld, W. Lerche, S. Samuel and S. Watamura, "Conformal Techniques, Bosonization And Tree Level String Amplitudes," Nucl. Phys. B 288, 173 (1987).

[59] K. Ohmori, "Open Superstring Field Theory Applied to Tachyon Condensation," PhD Thesis, Univ. of Tokyo (2004).

[60] I. Y. Arefeva, P. B. Medvedev and A. P. Zubarev, "Background Formalism For Superstring Field Theory," Phys. Lett. B 240, 356 (1990).

[61] B. V. Urosevic and A. P. Zubarev, "On The Component Analysis Of Modified Superstring Field Theory Actions," Phys. Lett. B 246 (1990) 391.

[62] J. Kluson, "Exact solutions in SFT and marginal deformation in BCFT," JHEP 0312, 050 (2003) arXiv:hep-th/0303199.

[63] A. Sen, "Open String Field Theory In Nontrivial Background Field: Gauge Invariant Action," Nucl. Phys. B 334, 350 (1990).

[64] J. Kluson, "Exact solutions in open bosonic string field theory and marginal deformation in CFT," Int. J. Mod. Phys. A 19, 4695 (2004) arXiv:hep-th/0209255. 